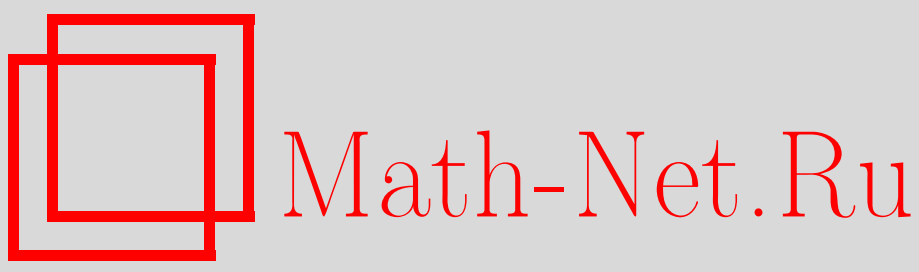

Е. Ю. Панов, Об одной аппроксимационной схеме для мерозначных решений квазилинейного уравнения первого порядка, Матем. сб., 1997, том 188, номер 1, 83-108

DOI: https://doi.org/10.4213/sm189

Использование Общероссийского математического портала Math-Net.Ru подразумевает, что вы прочитали и согласны с пользовательским соглашением http://www . mathnet.ru/rus/agreement

Параметры загрузки:

IP : 52.23 .180 .231

26 апреля 2023 г., $16: 45: 26$ 
УДК 517.95

\author{
Е.Ю. Панов
}

\title{
Об одной аппроксимационной схеме для мерозначных решений квазилинейного уравнения первого порядка
}

\footnotetext{
В работе приводится кинетическая формулировка мерозначных решений задачи Коши для квазилинейного уравнения первого порядка. С помощью специальной аппроксимации правой части соответствующего кинетического уравнения строится семейство уравнений, для которых доказывается однозначная разрешимость задачи Коши и сходимость (может быть после выделения подпоследовательности) получающейся последовательности решений к обобщенному решению исходной задачи.

Библиографой: 12 названий.
}

\section{Введение}

В полупространстве $(t, x) \in \Pi=(0,+\infty) \times \mathbb{R}^{n}$ рассмотрим квазилинейное уравнение первого порядка:

$$
\begin{gathered}
u_{t}+\operatorname{div}_{x} \varphi(u)=0, \\
u=u(t, x), \quad \varphi(u)=\left(\varphi_{1}(u), \ldots, \varphi_{n}(u)\right) \in\left(C^{1}(\mathbb{R})\right)^{n} .
\end{gathered}
$$

Нелокальная теория обобщенных решений (о.р.) задачи Коши для уравнения (1) с начальным условием

$$
u(0, x)=u_{0}(x) \in L^{\infty}\left(\mathbb{R}^{n}\right)
$$

была построена С.Н. Кружковым в работах [1]-[2]. Приведем

ОПРЕДЕЛЕНИЕ 1. Ограниченная измеримая функция $u=u(t, x)$ называется о.p. задачи Коши (1), (2), если:

a) $\forall k \in \mathbb{R}$

$$
|u-k|_{t}+\operatorname{div}_{x}[(\varphi(u)-\varphi(k)) \operatorname{sign}(u-k)] \leqslant 0
$$

в смысле распределений на П (в $\left.\mathscr{D}^{\prime}(\Pi)\right)$;

b) ess $\lim _{t \rightarrow 0} u(t, \cdot)=u_{0}$ в $L_{\text {loc }}^{1}\left(\mathbb{R}^{n}\right)$, т.е. существует множество нулевой меры Лебега $\mathscr{E} \subset(0,+\infty)$ такое, что для $t \notin \mathscr{E} u(t, \cdot) \in L_{\mathrm{loc}}^{1}\left(\mathbb{R}^{n}\right)$ и при $t \rightarrow 0$, $t \notin \mathscr{E}$

$$
u(t, \cdot) \rightarrow u_{0} \quad \text { в } \quad L_{\text {loc }}^{1}\left(\mathbb{R}^{n}\right) .
$$

Работа выполнена при финансовой поддержке INTAS (грант № 94-2187). 
Условие (3) означает, что для любой неотрицательной пробной функции $g=$ $g(t, x) \in C_{0}^{\infty}(\Pi)$

$$
\int_{\Pi}\left[|u-k| g_{t}+\sum_{i=1}^{n}\left(\varphi_{i}(u)-\varphi_{i}(k)\right) \operatorname{sign}(u-k) g_{x_{i}}\right] d t d x \geqslant 0 .
$$

Полагая в (3) $k= \pm\|u\|_{\infty}$, получим, что в $\mathscr{D}^{\prime}(\Pi) \pm\left\{u_{t}+\operatorname{div}_{x} \varphi(u)\right\} \leqslant 0$, т.е. $u_{t}+\operatorname{div}_{x} \varphi(u)=0$ и о.р. $u(t, x)$ удовлетворяет уравнению (1) в смысле распределений.

Известно (см. [1], [2]), что всегда существует единственное о.р. задачи (1), (2).

В работе [3] приведена кинетическая формулировка задачи (1), (2). А именно, показано, что функция $u=u(t, x)$ является о.р. задачи (1), (2) тогда и только тогда, когда функция $f=f(t, x, v)=\chi_{u(t, x)}(v)$, где при $u, v \in \mathbb{R}$

$$
\chi_{u}(v)=\theta(u-v)-\theta(-v), \quad \theta(\lambda)=\left\{\begin{array}{l}
1, \lambda>0, \\
0, \quad \lambda \leqslant 0,
\end{array}\right. \text { - функция Хевисайда, }
$$

является о.р. (в смысле распределений) соответствуюшей задачи для кинетического уравнения

$$
\frac{\partial}{\partial t} f+\left(\varphi^{\prime}(v), \nabla_{x} f\right)=\frac{\partial}{\partial v} m
$$

$\left((\cdot, \cdot)\right.$ - скалярное умножение на $\left.\mathbb{R}^{n}\right)$ с некоторой неотрицательной локально конечной мерой $m=m(t, x, v)$ на $\Pi \times \mathbb{R}$, финитной по переменной $v$, и начальным условием

$$
f(0, x, v)=f_{0}(x, v)
$$

где $f_{0}(x, v)=\chi_{u_{0}(x)}(v)$.

Начальное условие (5) понимается аналогично условию b) из определения 1 :

$$
\underset{t \rightarrow 0}{\operatorname{ess} \lim _{t \rightarrow 0}} f(t, \cdot, \cdot)=f_{0} \quad \text { в } \quad L_{\mathrm{loc}}^{1}\left(\mathbb{R}^{n+1}\right) .
$$

Кроме того, в [3] приведена следующая аппроксимационная схема. Рассмотрим при $\varepsilon>0$ решение $\left(\right.$ в $\left.\mathscr{D}^{\prime}(\Pi \times \mathbb{R})\right) f^{\varepsilon}=f^{\varepsilon}(t, x, v)$ задачи Коши для уравнения

$$
\begin{gathered}
\frac{\partial}{\partial t} f^{\varepsilon}+\left(\varphi^{\prime}(v), \nabla_{x} f^{\varepsilon}\right)=\frac{1}{\varepsilon}\left(\bar{f}^{\varepsilon}-f^{\varepsilon}\right) \\
\bar{f}^{\varepsilon}(t, x, v)=\chi_{\rho^{\varepsilon}(t, x)}(v), \quad \rho^{\varepsilon}(t, x)=\int_{\mathbb{R}} f^{\varepsilon}(t, x, v) d v
\end{gathered}
$$

с начальньм условием $f^{\varepsilon}(0, x, v)=f_{0}(x, v)$. Тогда, как показано в [3], при $\varepsilon \rightarrow 0$ $f^{\varepsilon}(t, x, v) \rightarrow f(t, x, v)$ в $L_{\text {loc }}^{1}(\Pi \times \mathbb{R})$, где $f(t, x, v)=\chi_{u(t, x)}(v)-$ o.p. задачи $(4),(5)$.

В настояшей работе мы распространяемприведенные выше результаты на более общий случай мерозначных решений. При этом для соответствующей аппроксимирующей задачи будет доказана однозначная разрешимость.

Напомним (см. [4], [5]), что мерозначной функцией на измеримом множестве $\Omega \subset \mathbb{R}^{m}$ (здесь $m \in \mathbb{N}$ произвольно) назьвается слабо измеримое отображение $x \mapsto \nu_{x}$ множества $\Omega$ в пространство $\operatorname{Prob}_{0}(\mathbb{R})$ вероятностных борелевских мер с компактным носителем на прямой $\mathbb{R}$. 
Слабая измеримость $\nu_{x}$ означает, что для любой непрерывной функции $p(\lambda)$ функция $x \mapsto \int p(\lambda) d \nu_{x}(\lambda)$ измерима на $\Omega$.

Мерозначная функция $\nu_{x}$ называется ограниченной, если существует $M>0$ такое, что для п.в. $x \in \Omega \operatorname{supp} \nu_{x} \subset[-M, M]$. Минимальное из таких $M$ будем обозначать $\left\|\nu_{x}\right\|_{\infty}$.

Наконец, мерозначные функции вида: $\nu_{x}(\lambda)=\delta(\lambda-u(x)), u(x) \in L^{\infty}(\Omega)$ $(\delta(\lambda-u)$ - мера Дирака в точке $u \in \mathbb{R})$ называются регулярными и будут нами отождествляться с соответствуюшими функциями $u(x)$. Таким образом, имеется естественное вложение $L^{\infty}(\Omega) \subset M V(\Omega)$, где $M V(\Omega)$ - множество ограниченных мерозначных функций на $\Omega$.

Рассмотрим мерозначные решения (м.р.) $\nu_{t, x} \in M V(\Pi)$ задачи Коши для уравнения (1) с начальным условием

$$
\nu_{0, x}=\nu_{x}^{0} \in M V\left(\mathbb{R}^{n}\right) .
$$

ОПРЕДЕЛЕНИЕ 2 (см. [5], [6]). Ограниченная мерозначная функция $\nu_{t, x}$ называется м.р. задачи (1), (7), если:

a) $\forall k \in \mathbb{R}$

$$
\frac{\partial}{\partial t} \int|\lambda-k| d \nu_{t, x}(\lambda)+\operatorname{div}_{x} \int(\varphi(\lambda)-\varphi(k)) \operatorname{sign}(\lambda-k) d \nu_{t, x}(\lambda) \leqslant 0 \quad \text { в } \quad \mathscr{D}^{\prime}(\Pi) ;
$$

b) $\forall p(\lambda) \in C(\mathbb{R})$

$$
\underset{t \rightarrow 0}{\operatorname{ess} \lim _{t \rightarrow}} \int p(\lambda) d \nu_{t, x}(\lambda)=\int p(\lambda) d \nu_{x}^{0}(\lambda) \text { в } \quad L_{\mathrm{loc}}^{1}\left(\mathbb{R}^{n}\right) .
$$

Заметим, что определение 2 согласуется с определением 1 в том смысле, что функция $u(t, x)$ является о.р. задачи $(1),(2)$ тогда и только тогда, когда она, как регулярная мерозначная функция $\nu_{t, x}(\lambda)=\delta(\lambda-u(t, x))$, является м.р. задачи $(1),(7)$ с регулярной начальной функцией $\nu_{x}^{0}(\lambda)=\delta\left(\lambda-u_{0}(x)\right)$.

Можно интерпретировать мерозначную функцию $\nu_{t, x}$ как случайное поле $u(t, x)$, где при фиксированном $(t, x) \in \Pi$ значение $u(t, x)$ - случайная величина с распределением $\nu_{t, x}$. Тогда требование (8) означает, что $u(t, x)$ удовлетворяет условию (3) "в среднем", т.е.

$$
\mathrm{E}(|u-k|)_{t}+\operatorname{div}_{x} \mathrm{E}((\varphi(u)-\varphi(k)) \operatorname{sign}(u-k)) \leqslant 0 \text { в } \mathscr{D}^{\prime}(\Pi),
$$

здесь $\mathrm{E}$ - знак математического ожидания.

Полагая в (8) $k= \pm M$, где $M=\left\|\nu_{t, x}\right\|_{\infty}$, получим, что

$$
\frac{\partial}{\partial t} \int \lambda d \nu_{t, x}(\lambda)+\operatorname{div}_{x} \int \varphi(\lambda) d \nu_{t, x}(\lambda)=0 \quad \text { в } \quad \mathscr{D}^{\prime}(\Pi),
$$

т.е. соответствуюшее случайное поле $u(t, x)$ удовлетворяет уравнению (1) "в среднем".

Известно (см. [7]-[9]), что м.р. задачи Коши (1), (7) всегда сушествует и заведомо неединственно, если начальная функция $\nu_{x}^{0}$ нерегулярна. Один из способов выделения класса сушествования и единственности предложен в [8] (подробно в [9]). 


\section{§1. Кинетическая интерпретация м.р.}

Пусть $\Omega \subset \mathbb{R}^{m}$ - измеримое множество, $\nu_{x} \in M V(\Omega), M=\left\|\nu_{x}\right\|_{\infty} ; f(x, v)=$ $F\left(\nu_{x}\right)(v)=\nu_{x}((v,+\infty))$ - функция распределения меры $\nu_{x}$. При фиксированном $x \in \Omega$ функция $f(x, v)$ не возрастает и непрерьвна справа по переменной $v \in \mathbb{R}$; для п.в. $x \in \Omega$ имеем $f(x, v)=0$ при $v \geqslant M, f(x, v)=1$ при $v<-M$. Кроме того, из слабой измеримости отображения $x \mapsto \nu_{x}$ легко следует (см., например, лемму 3 в [10]), что функция $f(x, v)$ измерима по совокупности переменных. Пусть $F_{M}(\Omega)$ - класс функций с указанными свойствами. Ясно, что включение $f(x, v) \in$ $F_{M}(\Omega)$ эквивалентно сушествованию мерозначной функции $\nu_{x} \in M V(\Omega)$ такой, что $\left\|\nu_{x}\right\|_{\infty} \leqslant M$ и $f(x, v)=\nu_{x}((v,+\infty))$.

Основным результатом параграфа является следующая

Теорема 1. Ограниченная мерозначная функиия $\nu_{t, x}$ является м.р. задачи (1), (7) тогда и только тогда, когда соответствующая функция распределения $f(t, x, v)=\nu_{t, x}((v,+\infty))$ является о.р. задачи (4), (5) с начальной функиией $f_{0}(x, v)=\nu_{x}^{0}((v,+\infty))$.

Для доказательства теоремы 1 нам понадобится ряд вспомогательных результатов.

Лемма 1. Пусть $\nu_{t, x}-$ м.р. задачи (1), (7). Тогдa $\forall v \in \mathbb{R}$

$$
\frac{\partial}{\partial t} \int|\lambda-v| d \nu_{t, x}(\lambda)+\operatorname{div}_{x} \int(\varphi(\lambda)-\varphi(v)) \operatorname{sign}(\lambda-v) d \nu_{t, x}(\lambda)=-\mu^{v}
$$

в $\mathscr{D}^{\prime}(\Pi)$, где $\mu^{v}$ - неотрицательная локально конечная мера на П. При этом, если $M=\left\|\nu_{t, x}\right\|_{\infty}$, то $\mu^{v}=0$ для $v \notin[-M, M]$; соответствие $v \mapsto \mu^{v}$ слабо непрерывно, т.е. $\forall g(t, x) \in C_{0}(\Pi)$ функция $v \mapsto \int g(t, x) d \mu^{v}(t, x)$ непрерывна.

ДокАЗАТЕЛЬСТво. При $v \in \mathbb{R}$ обозначим

$$
\begin{aligned}
& P=P(t, x)=\int|\lambda-v| d \nu_{t, x}(\lambda), \\
& Q=Q(t, x)=\int(\varphi(\lambda)-\varphi(v)) \operatorname{sign}(\lambda-v) d \nu_{t, x}(\lambda) \in \mathbb{R}^{n}
\end{aligned}
$$

и рассмотрим функционал

$$
L^{v}(g)=\int_{\Pi}\left[P g_{t}+\left(Q, \nabla_{x} g\right)\right] d t d x, \quad g=g(t, x) \in C_{0}^{\infty}(\Pi) .
$$

Из условия (8) следует неотрицательность $L^{v}: L^{v}(g) \geqslant 0$ при $g \geqslant 0$. Поэтому (подробности содержатся, например, в [10, лемма 5]), функционал $L^{v}$ непрерывен в топологии $C_{0}(\Pi)$ и однозначно продолжается до ограниченного неотрицательного функционала на $C_{0}(\Pi)$; по теореме Рисса-Маркова о представлении таких функционалов существует локально-конечная неотрицательная борелевская мера $\mu^{v}$ на П такая, что $L^{v}(g)=\int g(t, x) d \mu^{v}(t, x)$.

Учитьвая определение функционала $L^{v}$, получаем, что в $\mathscr{D}^{\prime}(\Pi)$

$$
P_{t}+\operatorname{div}_{x} Q=-\mu^{v} .
$$


Равенство (10) доказано.

Далее, если $v \notin[-M, M]$, то для п.в. $(t, x) \in \Pi$ имеем $|\lambda-v|=(v-\lambda) \operatorname{sign}(v)$, $(\varphi(\lambda)-\varphi(v)) \operatorname{sign}(\lambda-v)=(\varphi(v)-\varphi(\lambda)) \operatorname{sign}(v)$ на $\operatorname{supp} \nu_{t, x}$ и $\forall g(t, x) \in C_{0}^{\infty}(\Pi)$

$$
L^{v}(g)= \pm \int_{\Pi}\left[\left(\int \lambda d \nu_{t, x}(\lambda)\right) g_{t}+\left(\int \varphi(\lambda) d \nu_{t, x}(\lambda), \nabla_{x} g\right)\right] d t d x=0
$$

по равенству (9). Следовательно, $\mu^{v}=0$ при $v \notin[-M, M]$.

Для доказательства последнего утверждения леммы заметим, что при фиксированной функции $g(t, x) \in C_{0}^{\infty}(\Pi)$ величина $L^{v}(g)$ непрерывно зависит от $v \in \mathbb{R}$. Следовательно, функции $v \mapsto \int g(t, x) d \mu^{v}(t, x)$ непрерывны при $g(t, x) \in C_{0}^{\infty}(\Pi)$. Если $C \subset \Pi$ - компакт, $g(t, x) \in C_{0}^{\infty}(\Pi), g(t, x) \geqslant 0, g(t, x)=1$ при $(t, x) \in C$, то из непрерывности функции $v \mapsto \int g(t, x) d \mu^{v}(t, x)$ следует ограниченность значений $\mu^{v}(C)$ при $v \in \mathbb{R}\left(\right.$ с учетом того, что $\mu^{v}=0$ вне $\left.[-M, M]\right)$. Пусть $g(t, x) \in C_{0}(\Pi)$. Рассмотрим последовательность $g_{r}(t, x) \in C_{0}^{\infty}(\Pi), r \in \mathbb{N}$, сходящуюся равномерно к функции $g(t, x)$, такую, что для всех $r \in \mathbb{N} g_{r}=0$ вне некоторого компакта $C \subset$ П. Тогда с учетом ограниченности $\mu^{v}(C)$ последовательность непрерьвных по $v \in \mathbb{R}$ функций $\int_{C} g_{r}(t, x) d \mu^{v}(t, x)$ при $r \rightarrow \infty$ равномерно сходится к функции $\int g(t, x) d \mu^{v}(t, x)$. Поэтому предельная функция $v \mapsto \int g(t, x) d \mu^{v}(t, x)$ непрерывна и в силу произвольности $g(t, x) \in C_{0}(\Pi)$ отображение $v \mapsto \mu^{v}$ слабо непрерывно. Лемма полностью доказана.

Лемма 2. Пусть $h(v) \in C_{0}^{1}(\mathbb{R}), p(\lambda)=\int|\lambda-v| h^{\prime}(v) d v$,

$$
q_{i}(\lambda)=\int\left(\varphi_{i}(\lambda)-\varphi_{i}(v)\right) \operatorname{sign}(\lambda-v) h^{\prime}(v) d v, \quad i=1, \ldots, n
$$

Тогда $p(\lambda), q_{i}(\lambda) \in C^{1}(\mathbb{R}) u p^{\prime}(\lambda)=2 h(\lambda), q_{i}^{\prime}(\lambda)=\varphi_{i}^{\prime}(\lambda) p^{\prime}(\lambda)$.

ДокАЗАТЕЛЬСтво. В смысле распределений на $\mathbb{R}$

$$
\begin{aligned}
p^{\prime}(\lambda) & =\int|\lambda-v|_{\lambda}^{\prime} h^{\prime}(v) d v=\int \operatorname{sign}(\lambda-v) h^{\prime}(v) d v \\
& =\int_{-\infty}^{\lambda} h^{\prime}(v) d v-\int_{\lambda}^{+\infty} h^{\prime}(v) d v=2 h(\lambda) \\
q_{i}^{\prime}(\lambda) & =\int\left[\left(\varphi_{i}(\lambda)-\varphi_{i}(v)\right) \operatorname{sign}(\lambda-v)\right]_{\lambda}^{\prime} h^{\prime}(v) d v \\
& =\int \varphi_{i}^{\prime}(\lambda) \operatorname{sign}(\lambda-v) h^{\prime}(v) d v=\varphi_{i}^{\prime}(\lambda) \int \operatorname{sign}(\lambda-v) h^{\prime}(v) d v=\varphi_{i}^{\prime}(\lambda) p^{\prime}(\lambda) .
\end{aligned}
$$

Для завершения доказательства остается заметить, что $p^{\prime}(\lambda)=2 h(\lambda) \in C_{0}^{1}(\mathbb{R})$, $q_{i}^{\prime}(\lambda)=\varphi_{i}^{\prime}(\lambda) p^{\prime}(\lambda) \in C_{0}(\mathbb{R})$, следовательно, функции $p(\lambda), q_{i}(\lambda)$ дифференцируемы в классическом смысле и $p(\lambda), q_{i}(\lambda) \in C^{1}(\mathbb{R})$ (более того, $\left.p(\lambda) \in C^{2}(\mathbb{R})\right)$. 
Лемма 3. Пусть $\nu_{t, x} \in M V(\Pi), \nu_{x}^{0} \in M V\left(\mathbb{R}^{n}\right) ; f(t, x, v)=\nu_{t, x}((v,+\infty))$, $f_{0}(x, v)=\nu_{x}^{0}((v,+\infty))$. Тогда следующие условия әквивалентнь:

1) $\forall p(\lambda) \in C_{0}^{\infty}(\mathbb{R}) \quad \underset{t \rightarrow 0}{\operatorname{ess} \lim _{t \rightarrow 0}} \int p(\lambda) d \nu_{t, x}(\lambda)=\int p(\lambda) d \nu_{x}^{0}(\lambda)$ в $L_{\mathrm{loc}}^{1}\left(\mathbb{R}^{n}\right)$;

2) $\underset{t \rightarrow 0}{\operatorname{ess}} \lim _{t \rightarrow 0} f(t, x, v)=f_{0}(x, v)$ в $L_{\mathrm{loc}}^{1}\left(\mathbb{R}^{n+1}\right)$.

ДоказАтельство. Докажем сначала импликацию 2) $\Rightarrow 1$ ).

Пусть $M=\left\|\nu_{t, x}\right\|_{\infty}, p(\lambda) \in C_{0}^{\infty}(\mathbb{R}), h(v)=p^{\prime}(v)$. Из 2) следует, что

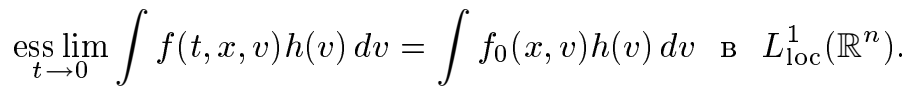

Интегрируя по частям, получим

$$
\int f(t, x, v) h(v) d v=\int f(t, x, v) d p(v)=-\int p(v) d f(t, x, v)=\int p(\lambda) d \nu_{t, x}(\lambda)
$$

и, аналогично, $\int f_{0}(x, v) h(v) d v=\int p(\lambda) d \nu_{x}^{0}(\lambda)$. Из этих равенств и (11) следует справедливость условия 1$)$.

Обратно, пусть выполнено 1). Тогда для любой функции $p=p(\lambda) \in C_{0}^{\infty}(\mathbb{R})$ сушествует множество нулевой меры $\mathscr{E}(p)$ такое, что при $t \rightarrow 0, t \notin \mathscr{E}(p)$,

$$
\int p(\lambda) d \nu_{t, x}(\lambda) \rightarrow \int p(\lambda) d \nu_{x}^{0}(\lambda) \quad \text { в } \quad L_{\mathrm{loc}}^{1}\left(\mathbb{R}^{n}\right)
$$

Выберем счетное всюду плотное множество $S \subset C_{0}^{\infty}(\mathbb{R})$ и положим $\mathscr{E}=\bigcup_{p \in S} \mathscr{E}(p)$. Тогда, по счетности $S, \mathscr{E}$ - множество нулевой меры и при $t \rightarrow 0, t \notin \mathscr{E}$, предельное соотношение (12) выполнено для всех $p \in S$. Пусть $\rho(x)=e^{-|x|}\left(|x|=(x, x)^{1 / 2}\right.$ евклидова норма на $\mathbb{R}^{n}$ ). Докажем, что при $t \rightarrow 0, t \notin \mathscr{E}$,

$$
\int_{\mathbb{R}^{n+1}}\left|f(t, x, v)-f_{0}(x, v)\right| \rho(x) d x d v \rightarrow 0
$$

откуда, очевидно, будет следовать 2) (заметим, что при $v \notin[-M, M]$ п.в. в $\mathbb{R}^{n}$ $\left.f(t, x, v)=f_{0}(x, v)\right)$.

Доказательство проведем методом от противного. Итак, предположим, что (13) ложно. Тогда существует $\delta>0$ и последовательность $t_{r}, r \in \mathbb{N}, t_{r} \notin \mathscr{E}$, $\lim _{r \rightarrow \infty} t_{r}=0$ такая, что

$$
\liminf _{r \rightarrow \infty} \int_{\mathbb{R}^{n+1}}\left|f\left(t_{r}, x, v\right)-f_{0}(x, v)\right| \rho(x) d x d v>\delta .
$$

Из (12) следует, что из последовательности $t_{r}$ можно выделить подпоследовательность (за которой мы сохраним прежнее обозначение $t_{r}$ ) такую, что

$$
\int p(\lambda) d \nu_{x}^{(r)}(\lambda) \underset{r \rightarrow \infty}{\longrightarrow} \int p(\lambda) d \nu_{x}^{0}(\lambda)
$$

п.в. в $\mathbb{R}^{n}$ при $\nu_{x}^{(r)}=\nu_{t_{r}, x}$. Применяя стандартную диагональную процедуру, мы можем выбрать указанную подпоследовательность общей для счетного множества 
функций $p(\lambda) \in S$. Тогда, так как $S$ плотно в $C_{0}^{\infty}(\mathbb{R})$, а, значит, и в $C_{0}(\mathbb{R})$, для п.в. $x \in \mathbb{R}^{n}$ имеем

$$
\int p(\lambda) d \nu_{x}^{(r)}(\lambda) \underset{r \rightarrow \infty}{\longrightarrow} \int p(\lambda) d \nu_{x}^{0}(\lambda)
$$

при всех $p(\lambda) \in C_{0}(\mathbb{R})$, т.е. последовательность мер $\nu_{x}^{(r)}$ сходится слабо к мере $\nu_{x}^{0}$. Из теоремы Хелли (см. [11, с. 209]) следует, что для таких $x \in \mathbb{R}^{n}$ соответствующая последовательность функций распределения $f\left(t_{r}, x, v\right)$ сходится к $f_{0}(x, v)$ во всех точках непрерьвности функции $v \mapsto f_{0}(x, v)$. Так как дополнение к множеству таких значений $v$ при каждом фиксированном $x \in \mathbb{R}^{n}$ не более чем счетно, то (очевидно, измеримое) множество $\left\{(x, v) \in \mathbb{R}^{n+1} \mid f\left(t_{r}, x, v\right) \underset{r \rightarrow \infty}{\longrightarrow} f_{0}(x, v)\right\}$ имеет полную меру Лебега. Итак, $f\left(t_{r}, x, v\right) \underset{r \rightarrow \infty}{\longrightarrow} f_{0}(x, v)$ п.в. в $\mathbb{R}^{n+1}, 0 \leqslant f \leqslant 1$, и по теореме Лебега об ограниченной сходимости

$$
\lim _{r \rightarrow \infty} \int_{\mathbb{R}^{n+1}}\left|f\left(t_{r}, x, v\right)-f_{0}(x, v)\right| \rho(x) d x d v=0
$$

что противоречит (14). Лемма доказана.

ДокаЗАТЕЛЬСтво теоРемы 1 . Пусть $h(v) \in C_{0}^{\infty}(\mathbb{R})$. Умножим равенство $(10)$ на $h^{\prime}(v)$ и проинтегрируем по $v \in \mathbb{R}$. Получим, что

$$
\frac{\partial}{\partial t} \int p(\lambda) d \nu_{t, x}(\lambda)+\operatorname{div}_{x} \int q(\lambda) d \nu_{t, x}(\lambda)=-\int_{\mathbb{R}} h^{\prime}(v) \mu^{v}(t, x) d v \quad \text { в } \quad \mathscr{D}^{\prime}(\Pi),
$$

где

$$
\begin{aligned}
& p(\lambda)=\int_{\mathbb{R}}|\lambda-v| h^{\prime}(v) d v \\
& q(\lambda)=\int_{\mathbb{R}}(\varphi(\lambda)-\varphi(v)) \operatorname{sign}(\lambda-v) h^{\prime}(v) d v=\left(q_{1}(\lambda), \ldots, q_{n}(\lambda)\right) \in \mathbb{R}^{n} .
\end{aligned}
$$

По лемме $2 p(\lambda) \in C^{1}(\mathbb{R}), q(\lambda) \in\left(C^{1}(\mathbb{R})\right)^{n}$ и $p^{\prime}(\lambda)=2 h(\lambda), q^{\prime}(\lambda)=2 \varphi^{\prime}(\lambda) h(\lambda)$. Отсюда и из формулы интегрирования по частям следует, что

$$
\begin{aligned}
& \int p(\lambda) d \nu_{t, x}(\lambda)=-\int p(\lambda) d_{\lambda} f(t, x, \lambda)=c_{0}+2 \int h(\lambda) f(t, x, \lambda) d \lambda, \\
& \int q(\lambda) d \nu_{t, x}(\lambda)=-\int q(\lambda) d_{\lambda} f(t, x, \lambda)=\boldsymbol{c}+2 \int h(\lambda) \varphi^{\prime}(\lambda) f(t, x, \lambda) d \lambda,
\end{aligned}
$$

где внеинтегральные члены

$$
c_{0}=-\left.\lim _{a \rightarrow+\infty} p(\lambda) f(t, x, \lambda)\right|_{-a} ^{a}, \quad c=-\left.\lim _{a \rightarrow+\infty} q(\lambda) f(t, x, \lambda)\right|_{-a} ^{a} \in \mathbb{R}^{n} .
$$

Так как при достаточно большом $a>0 f(t, x, a)=0, f(t, x,-a)=1$ и функции $p(-a), q_{i}(-a), i=1, \ldots, n$, постоянны (что вытекает из равенств $p^{\prime}(\lambda)=2 h(\lambda)$, $q^{\prime}(\lambda)=2 \varphi^{\prime}(\lambda) h(\lambda)$ и финитности $\left.h(\lambda)\right)$, то константы $c_{0}, \boldsymbol{c}=\left(c_{1}, \ldots, c_{n}\right)$ корректно 
определены и не зависят от $(t, x) \in$ П. Поэтому с учетом (16), (17) равенство (15) преобразуется к виду

$$
\begin{aligned}
\frac{\partial}{\partial t} \int h(\lambda) f(t, x, \lambda) d \lambda+\operatorname{div}_{x} \int h(\lambda) \varphi^{\prime}(\lambda) & f(t, x, \lambda) d \lambda \\
= & -\frac{1}{2} \int_{\mathbb{R}} h^{\prime}(v) \mu^{v}(t, x) d v \quad \text { в } \quad \mathscr{D}^{\prime}(\Pi) .
\end{aligned}
$$

Применяя это равенство к пробной функции $g(t, x) \in C_{0}^{\infty}(\Pi)$, получим, что

$$
\begin{aligned}
\int_{\Pi \times \mathbb{R}}\left[f(t, x, \lambda) h(\lambda) g_{t}(t, x)+f(t,\right. & \left.x, \lambda)\left(\varphi^{\prime}(\lambda), h(\lambda) \nabla_{x} g\right)\right] d t d x d \lambda \\
& =\frac{1}{2} \int_{\mathbb{R}} h^{\prime}(v)\left(\int_{\Pi} g(t, x) d \mu^{v}(t, x)\right) d v .
\end{aligned}
$$

Пусть $M=\left\|\nu_{t, x}\right\|_{\infty}$. Определим меру $m$ на $\Pi \times \mathbb{R}$ равенством

$$
\int_{\Pi \times \mathbb{R}} \xi(t, x, v) d m(t, x, v)=\frac{1}{2} \int_{\mathbb{R}}\left(\int_{\Pi} \xi(t, x, v) d \mu^{v}(t, x)\right) d v
$$

$\xi(t, x, v) \in C_{0}(\Pi \times \mathbb{R})$. По лемме 1 отображение $v \mapsto \mu^{v}$ слабо непрерывно и $\mu^{v}=0$ при $v \notin[-M, M]$, откуда легко следует непрерьвность и финитность функции $v \mapsto \int_{\Pi} \xi(t, x, v) d \mu^{v}(t, x)$, так что правая часть (19) корректно определена и задает неотрицательньй непрерьвньй функционал на пространстве функций $\xi \in C_{0}(\Pi \times \mathbb{R})$. По теореме Рисса-Маркова однозначно определена локально конечная мера $m \geqslant 0$ на $\Pi \times \mathbb{R}$, для которой вьполнено (19). Кроме того, ясно, что supp $m \subset \Pi \times[-M, M]$.

Пусть $\xi=\xi(t, x, v)=g(t, x) h(v)$. Тогда ввиду (18), (19) при $f=f(t, x, v)$

$$
\int_{\Pi \times \mathbb{R}}\left[f \xi_{t}+f\left(\varphi^{\prime}(v), \nabla_{x} \xi\right)\right] d t d x d v=\int_{\Pi \times \mathbb{R}} \xi_{v} d m(t, x, v) .
$$

Линейная оболочка функций $\xi(t, x, v)$ вида $g(t, x) h(v)$ плотна в $C_{0}^{\infty}(\Pi \times \mathbb{R})$ и по непрерывности последнее равенство верно для всех $\xi \in C_{0}^{\infty}(\Pi \times \mathbb{R})$, т.е.

$$
\frac{\partial}{\partial t} f+\left(\varphi^{\prime}(v), \nabla_{x} f\right)=\frac{\partial}{\partial v} m \text { в } \mathscr{D}^{\prime}(\Pi \times \mathbb{R}) .
$$

Таким образом, функция $f(t, x, v)$ удовлетворяет кинетическому уравнению (4). Из начального условия (7) в смысле определения 2 и леммы 3 следует, что

$$
\underset{t \rightarrow 0}{\operatorname{ess}} \lim _{t \rightarrow 0} f(t, x, v)=f_{0}(x, v) \quad \text { в } L_{\mathrm{loc}}^{1}\left(\mathbb{R}^{n+1}\right),
$$

т.е. вьполнено начальное условие (6).

Обратно, если $f(t, x, v) \in F_{M}(\Pi)$ - o.p. задачи Коши (4), (5), то из равенства (4) следует, что $\frac{\partial}{\partial v} m=0$ при $v \notin[-M, M]$ и ввиду финитности меры $m$ по переменной $v$ имеем supp $m \subset \Pi \times[-M, M]$. Применяя равенство (4) к пробной функции 
$g(t, x) p^{\prime}(v)$, где $g=g(t, x) \in C_{0}^{\infty}(\Pi), g(t, x) \geqslant 0 ; p(v) \in C_{0}^{\infty}(\mathbb{R}), p^{\prime \prime}(v) \geqslant 0$ при $v \in[-M, M]$, получим, что

$$
\begin{array}{r}
\int_{\Pi}\left[\left(\int_{\mathbb{R}} f(t, x, v) p^{\prime}(v) d v\right) g_{t}+\left(\int_{\mathbb{R}} f(t, x, v) \varphi^{\prime}(v) p^{\prime}(v) d v, \nabla_{x} g\right)\right] d t d x \\
=\int_{\Pi \times \mathbb{R}} g(t, x) p^{\prime \prime}(v) d m(t, x, v) \geqslant 0 .
\end{array}
$$

Аналогично (16), (17) из формулы интегрирования по частям выводятся равенства:

$$
\begin{aligned}
\int_{\mathbb{R}} f(t, x, v) p^{\prime}(v) d v & =\int p(\lambda) d \nu_{t, x}(\lambda), \\
\int_{\mathbb{R}} f(t, x, v) \varphi^{\prime}(v) p^{\prime}(v) d v & =\int q(\lambda) d \nu_{t, x}(\lambda),
\end{aligned}
$$

где $q(\lambda)=\left(q_{1}(\lambda), \ldots, q_{n}(\lambda)\right), q(\lambda)=\int_{-\infty}^{\lambda} \varphi^{\prime}(\sigma) p^{\prime}(\sigma) d \sigma$. Тогда из $(20)$

$$
\int_{\Pi}\left[\left(\int p(\lambda) d \nu_{t, x}(\lambda)\right) g_{t}+\left(\int q(\lambda) d \nu_{t, x}(\lambda), \nabla_{x} g\right)\right] d t d x \geqslant 0
$$

Пусть $p(\lambda)=\int_{\mathbb{R}}|\lambda-v| h^{\prime}(v) d v$, где $h(v) \in C_{0}^{\infty}(\mathbb{R}), h^{\prime}(v) \geqslant 0$ на $[-M, M]$. По лемме $2 p^{\prime \prime}(\lambda)=2 h^{\prime}(\lambda) \geqslant 0$ на $[-M, M]$ и, с точностью до постоянного вектора,

$$
q(\lambda)=\int_{\mathbb{R}}(\varphi(\lambda)-\varphi(v)) \operatorname{sign}(\lambda-v) h^{\prime}(v) d v
$$

Тогда, как следует из (21),

$$
\int_{\mathbb{R}} R(v) h^{\prime}(v) d v \geqslant 0
$$

где обозначено

$$
\begin{aligned}
& R(v)=\int_{\Pi}\left[\left(\int|\lambda-v| d \nu_{t, x}(\lambda)\right) g_{t}\right. \\
&\left.+\left(\int(\varphi(\lambda)-\varphi(v)) \operatorname{sign}(\lambda-v) d \nu_{t, x}(\lambda), \nabla_{x} g\right)\right] d t d x
\end{aligned}
$$

Легко видеть, что при $v \notin[-M, M]$

$$
R(v)=-\operatorname{sign} v \cdot c,
$$

где

$$
c=\text { const }=\int_{\Pi}\left[\left(\int \lambda d \nu_{t, x}(\lambda)\right) g_{t}+\left(\int \varphi(\lambda) d \nu_{t, x}(\lambda), \nabla_{x} g\right)\right] d t d x .
$$

Имеем

$$
\begin{aligned}
0 & \leqslant \int_{\mathbb{R}} R(v) h^{\prime}(v) d v=\int_{-M}^{M} R(v) h^{\prime}(v) d v+c\left(\int_{-\infty}^{-M} h^{\prime}(v) d v-\int_{M}^{+\infty} h^{\prime}(v) d v\right) \\
& =\int_{-M}^{M} R(v) h^{\prime}(v) d v+c(h(-M)+h(M)) .
\end{aligned}
$$


Рассматривая семейство постоянных на $[-M, M]$ функций $h$, выводим отсюда, что $c=0$ и, следовательно, $\int_{-M}^{M} R(v) h^{\prime}(v) d v \geqslant 0$, откуда, с учетом непрерьвности $R(v)$ и произвольности $h(v) \in C_{0}^{\infty}(\mathbb{R}), h^{\prime}(v) \geqslant 0$, следует неотрицательность функции $R(v)$ при $v \in[-M, M]$. Напомним, что при $v \notin[-M, M] R(v)= \pm c=0$. Итак, $\forall v \in \mathbb{R} R(v) \geqslant 0$, что означает выполнение условия (8) (в силу произвольности $\left.g=g(t, x) \in C_{0}^{\infty}(\Pi), g(t, x) \geqslant 0\right)$.

Далее, из начального условия (6) и леммы 3

$$
\forall p(\lambda) \in C_{0}^{\infty}(\mathbb{R}) \quad \underset{t \rightarrow 0}{\operatorname{ess} \lim _{t \rightarrow 0}} \int p(\lambda) d \nu_{t, x}(\lambda)=\int p(\lambda) d \nu_{x}^{0}(\lambda) \text { в } \quad L_{\mathrm{loc}}^{1}\left(\mathbb{R}^{n}\right) .
$$

Так как пространство $C_{0}^{\infty}(\mathbb{R})$ плотно в $C_{0}(\mathbb{R})$, то последнее предельное соотношение верно для всех $p(\lambda) \in C_{0}(\mathbb{R})$, т.е. выполнено начальное условие b) из определения 2. Итак, $\nu_{t, x}-$ м.p. задачи (1), (7). Теорема полностью доказана.

\section{§2. Аппроксимирующая задача}

Фиксируем некоторое $\mathrm{M}>0$ и положим для измеримой на $(-M, M)$ функции $h(v)$

$$
m_{h}(\lambda)=-M+\operatorname{mes}\{v \in(-M, M) \mid h(v)>\lambda\},
$$

mes - мера Лебега; пусть при $v \in(-M, M) h^{*}(v)=\inf \left\{\lambda \in \mathbb{R} \mid m_{h}(\lambda) \leqslant v\right\}$ - невозрастаюшая перестановка функции $h(v)$ (cм. [12, c. 332]). Хорошо известно, что $h^{*}(v)$ - невозрастаюшая непрерьвная справа функция, равноизмеримая с $h(v)$, т.е. $m_{h}(\lambda) \equiv m_{h^{*}}(\lambda)$. Из равноизмеримости функций $h^{*}$ и $h$ следует, что для борелевской функции $p(u)$ функции $p(h(v)), p\left(h^{*}(v)\right)$ суммируемы или нет на $(-M, M)$ одновременно и, если эти функции суммируемы, то (см. [12, с. 333])

$$
\int_{-M}^{M} p\left(h^{*}(v)\right) d v=\int_{-M}^{M} p(h(v)) d v .
$$

Нам потребуются некоторые свойства перестановок, собранные в следуюшей лемме.

Лемма 4. 1) Eсли $h(v) \in L^{1}((-M, M)), \chi(v)$ - невозрастающая на $(-M, M)$ функция, то

$$
\int_{-M}^{M}\left(h^{*}(v)-h(v)\right) \chi(v) d v \geqslant 0
$$

в частности, $\forall \lambda \in[-M, M] \int_{-M}^{\lambda}\left(h^{*}(v)-h(v)\right) d v \geqslant 0$.

2) Eсли $h_{1}, h_{2} \in L^{2}((-M, M))$, mo

$$
\int_{-M}^{M}\left(h_{1}^{*}(v)-h_{2}^{*}(v)\right)^{2} d v \leqslant \int_{-M}^{M}\left(h_{1}(v)-h_{2}(v)\right)^{2} d v .
$$

3) Если $h_{1}, h_{2} \in L^{\infty}((-M, M))$, то $\forall v \in(-M, M)$

$$
\left|h_{1}^{*}(v)-h_{2}^{*}(v)\right| \leqslant\left\|h_{1}-h_{2}\right\|_{\infty} .
$$


ДоказатЕЛьство. Свойство 1) доказано в [12, с. 334]. Для доказательства свойства 2) заметим, что

$$
\begin{aligned}
& \int_{-M}^{M}\left(h_{1}^{*}(v)-h_{2}^{*}(v)\right)^{2} d v \\
&=\int_{-M}^{M}\left(h_{1}^{*}(v)\right)^{2} d v+\int_{-M}^{M}\left(h_{2}^{*}(v)\right)^{2} d v-2 \int_{-M}^{M} h_{1}^{*}(v) h_{2}^{*}(v) d v .
\end{aligned}
$$

Из $(22)$ при $p(h)=h^{2}$ следуют равенства

$$
\int_{-M}^{M}\left(h_{i}^{*}(v)\right)^{2} d v=\int_{-M}^{M}\left(h_{i}(v)\right)^{2} d v, \quad i=1,2 .
$$

Далее, по теореме о двух перестановках из [12, с. 334]

$$
\int_{-M}^{M} h_{1}^{*}(v) h_{2}^{*}(v) d v \geqslant \int_{-M}^{M} h_{1}(v) h_{2}(v) d v
$$

Поэтому из (23)

$$
\begin{aligned}
& \int_{-M}^{M}\left(h_{1}^{*}(v)-h_{2}^{*}(v)\right)^{2} d v \\
& \quad \leqslant \int_{-M}^{M}\left(h_{1}(v)\right)^{2} d v+\int_{-M}^{M}\left(h_{2}(v)\right)^{2} d v-2 \int_{-M}^{M} h_{1}(v) h_{2}(v) d v \\
& \quad=\int_{-M}^{M}\left(h_{1}(v)-h_{2}(v)\right)^{2} d v,
\end{aligned}
$$

что доказывает 2).

Для доказательства 3) обозначим $c=\left\|h_{1}-h_{2}\right\|_{\infty}$. Тогда $h_{2}(v)-c \leqslant h_{1}(v) \leqslant$ $h_{2}(v)+c$ п.в. на $(-M, M)$. Далее, как непосредственно следует из определения невозрастаюшей перестановки, $f^{*}(v) \leqslant g^{*}(v)$ на $(-M, M)$, если $f(v) \leqslant g(v)$ п.в. на $(-M, M)$ и $(f+c)^{*}(v)=f^{*}(v)+c, c=$ const.

Используя эти свойства, получим неравенства

$$
h_{2}^{*}(v)-c \leqslant h_{1}^{*}(v) \leqslant h_{2}^{*}(v)+c,
$$

то есть

$$
\left|h_{1}^{*}(v)-h_{2}^{*}(v)\right| \leqslant c=\left\|h_{1}-h_{2}\right\|_{\infty} .
$$

Лемма полностью доказана.

Рассмотрим уравнение

$$
f_{t}+\left(\varphi^{\prime}(v), \nabla_{x} f\right)=r(g-f),
$$

$f=f(t, x, v) \in L^{\infty}(\Pi \times \mathbb{R}), r=$ const $>0$, нелинейный оператор $f \mapsto F f=g$ определяется равенством

$$
g=g(t, x, v)= \begin{cases}1, & v \leqslant-M, \\ 0, & v \geqslant M, \\ l\left(f^{*}(t, x ; v)\right), & v \in(-M, M),\end{cases}
$$


где $f^{*}(t, x ; v)$ - невозрастающая перестановка функции $v \mapsto f(t, x, v)$ по переменной $v \in(-M, M)$ (так как для п.в. $(t, x) \in \Pi$ функция $v \mapsto f(t, x, v)$ измерима, то перестановка $f^{*}(t, x ; v)$ корректно определена); $l(f)=\max (\min (f, 1), 0)$.

Пусть далее $\nu_{x}^{0} \in M V\left(\mathbb{R}^{n}\right),\left\|\nu_{x}^{0}\right\|_{\infty} \leqslant M, f_{0}(x, v)=\nu_{x}^{0}((v,+\infty)) \in F_{M}\left(\mathbb{R}^{n}\right)$.

Рассмотрим задачу Коши для уравнения $(24)$ с начальным условием

$$
f(0, x, v)=f_{0}(x, v),
$$

понимаемом в смысле предельного соотношения

$$
\underset{t \rightarrow 0}{\operatorname{ess} \lim _{t \rightarrow 0}} f(t, \cdot)=f_{0} \quad \text { в } \quad L_{\mathrm{loc}}^{1}\left(\mathbb{R}^{n+1}\right) .
$$

Мы будем рассматривать обобшенные решения (о.р.) $f(t, x, v) \in L^{\infty}(\Pi \times \mathbb{R})$ задачи $(24),(25)$, удовлетворяющие уравнению $(24)$ в $D^{\prime}(\Pi \times \mathbb{R})$ и начальному условию (25). Как будет показано в следуюшем параграфе, после возможного выделения подпоследовательности последовательность о.p. $f_{r}(t, x, v)$ задачи $(24),(25)$ (с параметром $r \in \mathbb{N}$ в правой части (24)) сходится при $r \rightarrow \infty$ к о.p. $f(t, x, v)$ задачи $(4),(5)$, определяя в силу теоремы 1 м.р. задачи $(1),(7)$ с мерозначной начальной функцией $\nu_{x}^{0}$.

В настоящем параграфе мы сосредоточимся на исследовании аппроксимирующей задачи $(24),(25)$. Верна

Лемма 5. Пусть $f(t, x, v)-$ o.p. задачи (24), (25). Тогда $0 \leqslant f(t, x, v) \leqslant 1$ n.в. на $\Pi \times \mathbb{R} ; f(t, x, v)=0$ для п.в. $(t, x, v) \in \Pi \times(M,+\infty), f(t, x, v)=1$ для n.в. $(t, x, v) \in \Pi \times(-\infty,-M)$.

ДокАЗАТЕЛЬСТво. В переменных $y=x-\varphi^{\prime}(v) t \in \mathbb{R}^{n}$ уравнение (24) перепишется в виде

$$
\bar{f}_{t}=r(\bar{g}-\bar{f}),
$$

где $\bar{f}=\bar{f}(t, y, v)=f\left(t, y+\varphi^{\prime}(v) t, v\right), \bar{g}=\bar{g}(t, y, v)=g\left(t, y+\varphi^{\prime}(v) t, v\right)$ и, так как при $v>M$ по построению $\bar{g}(t, y, v)=0$, то $\bar{f}_{t}+r \bar{f}=0$, откуда

$$
\bar{f}(t, y, v)=\bar{f}(0, y, v) e^{-r t}=f_{0}(y, v) e^{-r t}=0 \text { п.в. на } \Pi \times(M,+\infty)
$$

$\left(f_{0}(y, v) \in F_{M}\left(\mathbb{R}^{n}\right)\right.$ и, следовательно, $f_{0}(y, v)=0$ при $\left.v>M\right)$. При $v<-M$ $\bar{g}(t, y, v)=1$ и в силу $(26)(\bar{f}-1)_{t}+r(\bar{f}-1)=0, f_{0}(y, v)-1=0$ при $v<-M$, откуда $\bar{f}(t, y, v)-1=\left(f_{0}(y, v)-1\right) e^{-r t}=0$ п.в. на $\Pi \times(-\infty,-M)$. Далее, рассмотрим $C^{1}$-гладкую функцию

$$
\chi(u)= \begin{cases}u^{2}, & u \leqslant 0, \\ (u-1)^{2}, & u \geqslant 1, \\ 0, & u \in[0,1],\end{cases}
$$

и умножим равенство $(26)$ на $\chi^{\prime}(\bar{f})$. Это даст соотношение

$$
\frac{\partial}{\partial t} \chi(\bar{f})=r \chi^{\prime}(\bar{f})(\bar{g}-\bar{f})
$$

правая часть которого неположительна. Действительно, $\chi^{\prime}(\bar{f})=0$ при $\bar{f} \in[0,1]$; если $\bar{f}>1$, то $\chi^{\prime}(\bar{f})>0, \bar{g}-\bar{f}<0$ (так как $\bar{g} \leqslant 1$ ); если же $\bar{f}<0$, то $\chi^{\prime}(\bar{f})<0$, $\bar{g}-\bar{f}>0($ так как $\bar{g} \geqslant 0)$. 
Итак, $\frac{\partial}{\partial t} \chi(\bar{f}) \leqslant 0$, откуда п.в. на $\Pi \times \mathbb{R}$

$$
0 \leqslant \chi(\bar{f}(t, y, v)) \leqslant \chi\left(f_{0}(y, v)\right)=0
$$

(поскольку $\left.0 \leqslant f_{0}(y, v) \leqslant 1\right)$.

Это означает, что $0 \leqslant \bar{f}(t, y, v) \leqslant 1$ для п.в. $(t, y, v) \in \Pi \times \mathbb{R}$, и поэтому $0 \leqslant f(t, x, v) \leqslant 1$ п.в. на $\Pi \times \mathbb{R}$.

Лемма доказана.

ЗАмЕчАНИЕ 1 . Из леммы 5 следует, что для п.в. $(t, x) \in \Pi$ при $v \in(-M, M)$ $0 \leqslant f^{*}(t, x ; v) \leqslant 1$ и $g(t, x, v)=l\left(f^{*}(t, x ; v)\right)=f^{*}(t, x ; v)$.

Теорема 2. Пусть $f_{1}(t, x, v), f_{2}(t, x, v)$ - о.р. задачи $(24),(25)$ с начальнымми функииями $f_{1}^{0}(x, v), f_{2}^{0}(x, v) \in F_{M}\left(\mathbb{R}^{n}\right)$, соответственно $; N=\max _{v \in[-M, M]}\left|\varphi^{\prime}(v)\right|$,
$\rho(x)=e^{-|x|}, x \in \mathbb{R}^{n}$. Тогда для п.в. $t \in(0,+\infty)$

$$
\begin{aligned}
\int_{\mathbb{R}^{n+1}} & \left(f_{1}(t, x, v)-f_{2}(t, x, v)\right)^{2} \rho(x) d x d v \\
\leqslant & e^{N t} \int_{\mathbb{R}^{n+1}}\left(f_{1}^{0}(x, v)-f_{2}^{0}(x, v)\right)^{2} \rho(x) d x d v \\
= & e^{N t} \int_{\mathbb{R}^{n} \times[-M, M]}\left(f_{1}^{0}(x, v)-f_{2}^{0}(x, v)\right)^{2} \rho(x) d x d v
\end{aligned}
$$

ДоказАТЕЛЬСтво. Так как $f_{1}=f_{1}(t, x, v), f_{2}=f_{2}(t, x, v)-$ о.р. задачи $(24),(25)$, то в $D^{\prime}(\Pi \times \mathbb{R})$

$$
\frac{\partial}{\partial t} f_{1}+\left(\varphi^{\prime}(v), \nabla_{x} f_{1}\right)=r\left(g_{1}-f_{1}\right), \quad \frac{\partial}{\partial t} f_{2}+\left(\varphi^{\prime}(v), \nabla_{x} f_{2}\right)=r\left(g_{2}-f_{2}\right),
$$

здесь $g_{1}=F f_{1}, g_{2}=F f_{2}$. Вычитая второе равенство из первого, получим, что

$$
\frac{\partial}{\partial t}\left(f_{1}-f_{2}\right)+\left(\varphi^{\prime}(v), \nabla_{x}\left(f_{1}-f_{2}\right)\right)=r\left(\left(g_{1}-g_{2}\right)-\left(f_{1}-f_{2}\right)\right)
$$

Отсюда, после умножения на $2\left(f_{1}-f_{2}\right)$, следует равенство

$$
\frac{\partial}{\partial t}\left(f_{1}-f_{2}\right)^{2}+\left(\varphi^{\prime}(v), \nabla_{x}\left(f_{1}-f_{2}\right)^{2}\right)=2 r\left(\left(g_{1}-g_{2}\right)\left(f_{1}-f_{2}\right)-\left(f_{1}-f_{2}\right)^{2}\right)
$$

Пусть $\beta(s) \in C_{0}^{\infty}(\mathbb{R}), \beta(s) \geqslant 0, \operatorname{supp} \beta \subset[0,1], \int \beta(s) d s=1$.

При $\nu \in \mathbb{N}$ положим $\delta_{\nu}(s)=\nu \beta(\nu s)$ (ясно, что при $\nu \rightarrow \infty$ последовательность $\delta_{\nu}$ сходится в $D^{\prime}(\mathbb{R})$ к $\delta$-функции Дирака); $\alpha_{\nu}(t)=\int_{-\infty}^{t} \delta_{\nu}(s) d s, \chi_{\nu}(t)=$ $\alpha_{\nu}\left(t-t_{0}\right)-\alpha_{\nu}\left(t-t_{1}\right)$, где $t_{0}, t_{1}>0, t_{1}>t_{0}$. Очевидно, $0 \leqslant \chi_{\nu}(t) \leqslant 1$ и при $\nu \rightarrow \infty$ последовательность $\chi_{\nu}(t)$ поточечно сходится к характеристической функции интервала $\left(t_{0}, t_{1}\right]$. Пусть $h(t, x)=\exp (-N t-|x|)=e^{-N t} \rho(x)$. Умножим $(28)$ 
на $h(t, x) \chi_{\nu}(t)$ и проинтегрируем по $(t, x, v) \in \Pi \times \mathbb{R}$. Перебросив производные на пробную функцию $h(t, x) \chi_{\nu}(t)$, получим равенство

$$
\begin{aligned}
\int_{\Pi \times \mathbb{R}}\left(f_{1}-f_{2}\right)^{2} h(t, x)\left(\delta_{\nu}\left(t-t_{0}\right)-\delta_{\nu}\left(t-t_{1}\right)\right) d t d x d v \\
-\int_{\Pi \times \mathbb{R}}\left(f_{1}-f_{2}\right)^{2}\left[N+\left(\varphi^{\prime}(v), \frac{x}{|x|}\right)\right] \exp (-N t-|x|) \chi_{\nu}(t) d t d x d v \\
\quad=-2 r \int_{\Pi}\left[\int_{\mathbb{R}}\left(g_{1}-g_{2}\right)\left(f_{1}-f_{2}\right) d v-\int_{\mathbb{R}}\left(f_{1}-f_{2}\right)^{2} d v\right] h(t, x) \chi_{\nu}(t) d t d x
\end{aligned}
$$

Поскольку

$$
\left|\left(\varphi^{\prime}(v), \frac{x}{|x|}\right)\right| \leqslant\left|\varphi^{\prime}(v)\right| \leqslant N
$$

при $v \in[-M, M]$ и, как следует из леммы $5, f_{1}-f_{2}=0$ для п.в. $(t, x, v) \in$ $\Pi \times(\mathbb{R} \backslash[-M, M])$, то второй интеграл в левой части $(29)$ неотрицателен. Далее, $g_{1}=g_{2}$ при $v \notin[-M, M]$ и по неравенству Гёльдера

$$
\begin{aligned}
\int_{\mathbb{R}}\left(g_{1}-g_{2}\right)\left(f_{1}-f_{2}\right) d v & =\int_{-M}^{M}\left(g_{1}-g_{2}\right)\left(f_{1}-f_{2}\right) d v \\
& \leqslant\left(\int_{-M}^{M}\left(g_{1}-g_{2}\right)^{2} d v\right)^{1 / 2}\left(\int_{-M}^{M}\left(f_{1}-f_{2}\right)^{2} d v\right)^{1 / 2} \\
& \leqslant \int_{-M}^{M}\left(f_{1}-f_{2}\right)^{2} d v
\end{aligned}
$$

для п.в. $(t, x) \in \Pi$. Действительно, по замечанию 1 для п.в. $(t, x) \in \Pi$ при $v \in[-M, M] \quad g_{1}(t, x, v)=f_{1}^{*}(t, x ; v), g_{2}(t, x, v)=f_{2}^{*}(t, x ; v)$ и по свойству 2 из леммы 4

$$
\int_{-M}^{M}\left(g_{1}(t, x, v)-g_{2}(t, x, v)\right)^{2} d v \leqslant \int_{-M}^{M}\left(f_{1}(t, x, v)-f_{2}(t, x, v)\right)^{2} d v .
$$

Из (30) следует, что правая часть (29) также неотрицательна. В итоге, из (29) вытекает, что

$$
\int_{\Pi \times \mathbb{R}}\left(f_{1}-f_{2}\right)^{2} h(t, x)\left(\delta_{\nu}\left(t-t_{0}\right)-\delta_{\nu}\left(t-t_{1}\right)\right) d t d x d v \geqslant 0
$$

или

$$
\begin{aligned}
\int_{0}^{+\infty} & \left(\int_{\mathbb{R}^{n+1}}\left(f_{1}(t, x, v)-f_{2}(t, x, v)\right)^{2} h(t, x) d x d v\right) \delta_{\nu}\left(t-t_{1}\right) d t \\
& \leqslant \int_{0}^{+\infty}\left(\int_{\mathbb{R}^{n+1}}\left(f_{1}(t, x, v)-f_{2}(t, x, v)\right)^{2} h(t, x) d x d v\right) \delta_{\nu}\left(t-t_{0}\right) d t
\end{aligned}
$$

Пусть $E \subset(0,+\infty)$ - множество точек Лебега функции

$$
t \mapsto \int_{\mathbb{R}^{n+1}}\left(f_{1}(t, x, v)-f_{2}(t, x, v)\right)^{2} h(t, x) d x d v .
$$


Тогда $E$-множество полной меры (см. [11, с. 237]) и при $t_{0}, t_{1} \in E$ из (31) в пределе при $\nu \rightarrow \infty$ вытекает оценка

$$
\begin{aligned}
& \int_{\mathbb{R}^{n+1}}\left(f_{1}\left(t_{1}, x, v\right)-f_{2}\left(t_{1}, x, v\right)\right)^{2} h\left(t_{1}, x\right) d x d v \\
& \leqslant \int_{\mathbb{R}^{n+1}}\left(f_{1}\left(t_{0}, x, v\right)-f_{2}\left(t_{0}, x, v\right)\right)^{2} h\left(t_{0}, x\right) d x d v .
\end{aligned}
$$

Пусть $\mathscr{E} \subset(0,+\infty)$ - множество нулевой меры такое, что при $t \notin \mathscr{E}$ в соответствии с леммой 5 и начальным условием $(25) f_{1}(t, x, v), f_{2}(t, x, v) \in[0,1]$ для п.в. $(x, v) \in \mathbb{R}^{n+1}, f_{1}(t, x, v)=f_{2}(t, x, v)$ для п.в. $(x, v) \in \mathbb{R}^{n} \times(\mathbb{R} \backslash[-M, M])$ и при $t \rightarrow 0, t \notin \mathscr{E}, f_{1}(t, \cdot) \rightarrow f_{1}^{0}, f_{2}(t, \cdot) \rightarrow f_{2}^{0}$ в $L_{\mathrm{loc}}^{1}\left(\mathbb{R}^{n+1}\right)$.

Тогда при $t_{0} \rightarrow 0, t_{0} \in E \backslash \mathscr{E}$,

$$
\begin{array}{rl}
\int_{\mathbb{R}^{n+1}}\left(f_{1}\left(t_{0}, x, v\right)-f_{2}\left(t_{0}, x, v\right)\right)^{2} & h\left(t_{0}, x\right) d x d v \\
& \rightarrow \int_{\mathbb{R}^{n+1}}\left(f_{1}^{0}(x, v)-f_{2}^{0}(x, v)\right)^{2} h(0, x) d x d v
\end{array}
$$

и из (32) следует (с заменой $t_{1}$ на $\left.t\right)$, что $\forall t \in E$

$$
\begin{aligned}
\int_{\mathbb{R}^{n+1}}\left(f_{1}(t, x, v)-f_{2}(t, x, v)\right)^{2} h(t, x) d x d v & \\
& \leqslant \int_{\mathbb{R}^{n+1}}\left(f_{1}^{0}(x, v)-f_{2}^{0}(x, v)\right)^{2} h(0, x) d x d v .
\end{aligned}
$$

Для завершения доказательства остается заметить, что $h(t, x)=e^{-N t} \rho(x)$, $h(0, x)=\rho(x)$. Ясно также, что $f_{1}^{0}(x, v)-f_{2}^{0}(x, v)=0$ при $v \notin[-M, M]$. Теорема доказана.

СлЕДСТВИЕ 1. Обобщенное решение $f(t, x, v)$ задачи $(24),(25)$ единственно.

Для доказательства применим теорему 2 при $f_{1}^{0}=f_{2}^{0}=f_{0}$.

СлеДСТвИЕ 2. Пусть

$$
\omega_{2}^{x}(\sigma)=\sup _{\Delta x \in \mathbb{R}^{n},|\Delta x| \leqslant \sigma} \int_{\mathbb{R}^{n} \times[-M, M]}\left(f_{0}(x+\Delta x, v)-f_{0}(x, v)\right)^{2} \rho(x) d x d v
$$

- квадрат модуля непрерывности начальной функиии $f_{0}(x, v)$ по $x$ в $L^{2}\left(\mathbb{R}^{n} \times[-M, M], \rho(x) d x d v\right)$. Тогда для п.в. $t \in(0,+\infty)$

$$
\int_{\mathbb{R}^{n+1}}(f(t, x+\Delta x, v)-f(t, x, v))^{2} \rho(x) d x d v \leqslant e^{N t} \omega_{2}^{x}(|\Delta x|) .
$$

ДоказАтельство. Достаточно заметить, что при $\Delta x \in \mathbb{R}^{n}$ функция $f(t, x+\Delta x, v)$ является о.р. задачи $(24),(25)$ с начальной функцией $f_{0}(x+\Delta x, v)$ и применить теорему 2 при $f_{1}=f(t, x, v), f_{2}=f(t, x+\Delta x, v)$. 
ТЕОРЕма 3. Обобщенное решение задачи (24), (25) существует.

ДокаЗАТЕЛЬство. При $(t, x, v) \in \Pi \times \mathbb{R}$ пусть $y=x-\varphi^{\prime}(v) t$. Тогда замена $Q(t, x, v)=(t, y, v)$ является гомеоморфизмомпространства $\Pi \times \mathbb{R}$ в себя, обратное отображение $Q^{-1}$ задается формулой $x=y+\varphi^{\prime}(v) t$. Поскольку при фиксированных $t, v$ отображение $Q$ является сдвигом вектора $x \in \mathbb{R}^{n}$, это отображение (вместе с $Q^{-1}$ ) сохраняет меру Лебега, т.е. множество $A \subset \Pi \times \mathbb{R}$ измеримо по Лебегу одновременно с множеством $Q(A) \subset \Pi \times \mathbb{R}$ и $\operatorname{mes}(A)=\operatorname{mes}(Q(A))$. Для произвольной функции $h(t, x, v)$ на $\Pi \times \mathbb{R}$ будем обозначать $\bar{h}(t, y, v)=\left(h \circ Q^{-1}\right)(t, y, v)=$ $h\left(t, y+\varphi^{\prime}(v) t, v\right)$ (заметим, что функции $h, \bar{h}$ измеримы или нет по Лебегу одновременно).

Если $f(t, x, v)$ - о.р. задачи $(24),(25), g=F f=g(t, x, v)$, то по (26) (в смысле распределений)

$$
\frac{\partial}{\partial t} \bar{f}(t, y, v)+r \bar{f}(t, y, v)=r \bar{g}(t, y, v) \quad \text { и } \bar{f}(0, y, v)=f_{0}(y, v) .
$$

Из известной формулы для решения линейного обыкновенного дифференциального уравнения первого порядка следует, что задача (24), (25) эквивалентна интегральному уравнению

$$
\bar{f}(t, y, v)=e^{-r t} f_{0}(y, v)+r \int_{0}^{t} e^{-r(t-\tau)} \bar{g}(\tau, y, v) d \tau
$$

Решение этого уравнения естественно искать методом последовательных приближений. Определим последовательность $f_{m}(t, x, v), m \in \mathbb{N}$, такую, что соответствующие функции $\bar{f}_{m}(t, y, v)$ удовлетворяют условиям: $\bar{f}_{1}(t, y, v)=f_{0}(y, v)$,

$$
\bar{f}_{m+1}(t, y, v)=e^{-r t} f_{0}(y, v)+r \int_{0}^{t} e^{-r(t-\tau)} \bar{g}_{m}(\tau, y, v) d \tau
$$

где $g_{m}=F f_{m}$. Пусть $E=\bigcap_{m \in \mathbb{N}} E_{m}$, где $E_{m}=\left\{(y, v) \in \mathbb{R}^{n+1} \mid \bar{g}_{m}(\tau, y, v)\right.$ - измеримая по $\tau \in(0,+\infty)$ функция $\}$; тогда $E$ - множество полной меры и при $(y, v) \in E$ равенство (34) имеет смысл при всех $t \geqslant 0, m \in \mathbb{N}$.

Так как

$$
r \int_{0}^{t} e^{-r(t-\tau)} d \tau=\left(1-e^{-r t}\right), \quad 0 \leqslant \bar{g}_{m} \leqslant 1, \quad 0 \leqslant f_{0} \leqslant 1
$$

то из (34) $\forall m \in \mathbb{N} 0 \leqslant \bar{f}_{m} \leqslant 1$. Ясно также, что $\mathbb{R}^{n} \times(\mathbb{R} \backslash[-M, M]) \subset E$ и $\bar{f}_{m}(t, y, v)=0$ при $v>M, \bar{f}_{m}(t, y, v)=1$ при $v<-M$.

Обозначим при $m \in \mathbb{N}$

$$
\Delta_{m}(t)=\left\|\bar{f}_{m+1}(t, \cdot)-\bar{f}_{m}(t, \cdot)\right\|_{\infty}=\operatorname{essiup}_{(y, v) \in \mathbb{R}^{n+1}}\left|\bar{f}_{m+1}(t, y, v)-\bar{f}_{m}(t, y, v)\right| .
$$

Из (34) вытекает равенство

$$
\bar{f}_{m+2}(t, y, v)-\bar{f}_{m+1}(t, y, v)=r \int_{0}^{t} e^{-r(t-\tau)}\left(\bar{g}_{m+1}(\tau, y, v)-\bar{g}_{m}(\tau, y, v)\right) d \tau
$$


Оценим подынтегральное выражение в (35). С учетом определения функций $g_{m}$ и свойства 3$)$ из леммы 4 , при фиксированных $(\tau, v)$ на множестве полной меры значений $y \in \mathbb{R}^{n}$, для которых функции $u \mapsto f_{m}\left(\tau, y+\varphi^{\prime}(v) \tau, u\right)$ измеримы,

$$
\begin{aligned}
& \left|\bar{g}_{m+1}(\tau, y, v)-\bar{g}_{m}(\tau, y, v)\right| \\
& \quad=\left|g_{m+1}\left(\tau, y+\varphi^{\prime}(v) \tau, v\right)-g_{m}\left(\tau, y+\varphi^{\prime}(v) \tau, v\right)\right| \\
& \quad \leqslant\left|f_{m+1}^{*}\left(\tau, y+\varphi^{\prime}(v) \tau ; v\right)-f_{m}^{*}\left(\tau, y+\varphi^{\prime}(v) \tau ; v\right)\right| \\
& \quad \leqslant \operatorname{ess~sup}_{u \in[-M, M]}\left|f_{m+1}\left(\tau, y+\varphi^{\prime}(v) \tau, u\right)-f_{m}\left(\tau, y+\varphi^{\prime}(v) \tau, u\right)\right| \\
& \quad=\operatorname{esssup}_{u \in[-M, M]}\left|\bar{f}_{m+1}\left(\tau, y+\left(\varphi^{\prime}(v)-\varphi^{\prime}(u)\right) \tau, u\right)-\bar{f}_{m}\left(\tau, y+\left(\varphi^{\prime}(v)-\varphi^{\prime}(u)\right) \tau, u\right)\right|,
\end{aligned}
$$

откуда

$$
\begin{aligned}
& \underset{y \in \mathbb{R}^{n}}{\operatorname{ess} \sup }\left|\bar{g}_{m+1}(\tau, y, v)-\bar{g}_{m}(\tau, y, v)\right| \\
& \quad \leqslant \operatorname{ess~sup}_{(y, u) \in \mathbb{R}^{n+1}}\left|\bar{f}_{m+1}\left(\tau, y+\left(\varphi^{\prime}(v)-\varphi^{\prime}(u)\right) \tau, u\right)-\bar{f}_{m}\left(\tau, y+\left(\varphi^{\prime}(v)-\varphi^{\prime}(u)\right) \tau, u\right)\right| \\
& \quad=\operatorname{ess~sup}_{(z, u) \in \mathbb{R}^{n+1}}\left|\bar{f}_{m+1}(\tau, z, u)-\bar{f}_{m}(\tau, z, u)\right| .
\end{aligned}
$$

В последнем равенстве используется тот факт, что при фиксированных $\tau, v$ преобразование $(y, u) \mapsto(z, u)$ сохраняет меру Лебега. Из (36) следует, что

$$
\left\|\bar{g}_{m+1}(\tau, \cdot)-\bar{g}_{m}(\tau, \cdot)\right\|_{\infty} \leqslant \Delta_{m}(\tau),
$$

и из $(35)$

$$
\Delta_{m+1}(t)=r \int_{0}^{t} e^{-r(t-\tau)} \Delta_{m}(\tau) d \tau \leqslant r \int_{0}^{t} \Delta_{m}(\tau) d \tau .
$$

Ясно, что $\Delta_{1}(t) \leqslant 1$. Индукцией по $m \in \mathbb{N}$ из $(37)$ выводится оценка

$$
\Delta_{m}(t) \leqslant \frac{(r t)^{m-1}}{(m-1) !} .
$$

Тогда

$$
\sum_{m=1}^{\infty}\left\|\bar{f}_{m+1}(t, \cdot)-\bar{f}_{m}(t, \cdot)\right\|_{\infty} \leqslant \sum_{m=1}^{\infty} \frac{(r t)^{m-1}}{(m-1) !}=e^{r t}<\infty,
$$

что означает сходимость последовательности $f_{m}(t, y, v)$ в $L^{\infty}\left(\Pi_{T} \times \mathbb{R}\right)$, где $\Pi_{T}=[0, T] \times \mathbb{R}^{n}, T>0$ произвольно. Пусть $\bar{f}(t, y, v)$ - предельная функция $\bar{g}(t, y, v)=g\left(t, y+\varphi^{\prime}(v) t, v\right), g=F f$.

Аналогично (36) доказьвается оценка

$$
\left\|\bar{g}_{m}(t, \cdot)-\bar{g}(t, \cdot)\right\|_{\infty} \leqslant\left\|\bar{f}_{m}(t, \cdot)-\bar{f}(t, \cdot)\right\|_{\infty}
$$

из которой следует сходимость $\bar{g}_{m} \underset{m \rightarrow \infty}{\longrightarrow} \bar{g}_{\text {в }} L^{\infty}\left(\Pi_{T} \times \mathbb{R}\right)$. Переходя к пределу в (34) при $m \rightarrow \infty$, получим тогда, что для предельных функций $\bar{f}(t, y, v), \bar{g}(t, y, v)$ вьполнено (33). Это значит, что функция $f(t, x, v)=\bar{f}\left(t, x-\varphi^{\prime}(v) t, v\right)$ является о.р. задачи (24), (25). Теорема доказана.

Ниже нам понадобится следуюший результат. 
Tеорема 4. Пусть $f(t, x, v)-$ o.p. задачи (24), (25). Тогда для n.в. $\Delta t>0$

$$
\int_{\mathbb{R}^{n+1}}\left(f(\Delta t, x, v)-f_{0}(x, v)\right)^{2} \rho(x) d x d v \leqslant \omega^{t}(\Delta t),
$$

əде $\omega^{t}(\Delta t)=c \inf _{0<h<1}\left(\frac{\Delta t}{h}+\omega_{1}^{x}(h)\right)$,

$$
\omega_{1}^{x}(h)=\sup _{\Delta x \in \mathbb{R}^{n},|\Delta x| \leqslant h} \int_{\mathbb{R}^{n+1}}\left|f_{0}(x+\Delta x, v)-f_{0}(x, v)\right| \rho(x) d x d v
$$

- модуль непрерывности начальной функции по переменной $x$ в $L^{1}\left(\mathbb{R}^{n} \times[-M, M], \rho(x) d x d v\right) ; c-$ константа, не зависящая от $\Delta t, r$.

ДокАЗАТЕЛЬСТво. При $R>0$ обозначим $B_{R}$ - шар $\left\{x \in \mathbb{R}^{n}|| x \mid \leqslant R\right\}$. Предположим, что $\gamma(x) \in C_{0}^{\infty}\left(\mathbb{R}^{n}\right), \gamma(x) \geqslant 0, \operatorname{supp} \gamma \subset B_{1}, \int_{\mathbb{R}^{n}} \gamma(x) d x=1$. Пусть при $h>0 \gamma_{h}(x)=h^{-n} \gamma\left(h^{-1} x\right)$, так что при $h \rightarrow 0 \gamma_{h}(x)$ сходится в $D^{\prime}\left(\mathbb{R}^{n}\right)$ к $\delta$-функции Дирака. Фиксируем $t_{0}, t_{1}>0$ и положим при $\nu \in \mathbb{N} \chi_{\nu}(t)=\alpha_{\nu}\left(t-t_{0}\right)-$ $\alpha_{\nu}\left(t-t_{1}\right)$ (см. доказательство теоремы 2$)$. Рассмотрим также функцию

$$
\beta_{h}(x, v)=f_{0}(\cdot, v) * \gamma_{h}=\int f_{0}(x-y, v) \gamma_{h}(y) d y
$$

получаемую усреднением начальной функции $f_{0}$ по пространственным переменньм. По свойствам средних функций при $h \rightarrow 0 \beta_{h}(x, v) \rightarrow f_{0}(x, v)$ п.в. на $\mathbb{R}^{n+1}$ и при фиксированном $v \in \mathbb{R} \beta_{h}(x, v) \in C^{\infty}\left(\mathbb{R}^{n}\right)$. Так как

$$
\frac{\partial}{\partial x_{i}} \beta_{h}(x, v)=f_{0}(\cdot, v) * \frac{\partial}{\partial x_{i}} \gamma_{h}, \quad i=1, \ldots, n,
$$

TO

$$
\left|\nabla_{x} \beta_{h}(x, v)\right|=\left(\sum_{i=1}^{n}\left(\frac{\partial}{\partial x_{i}} \beta_{h}(x, v)\right)^{2}\right)^{1 / 2} \leqslant\left\|f_{0}(\cdot, v)\right\|_{\infty} \cdot\left\|\nabla_{x} \gamma_{h}\right\|_{1} \leqslant \frac{c_{1}}{h},
$$

$c_{1}=\left\|\nabla_{x} \gamma\right\|_{1}=$ const.

Положим $w(t, x, v)=\beta_{h}(x, v) \chi_{\nu}(t) \rho(x) \xi(v)$, где $\xi(v)-$ характеристическая функция отрезка $[-M, M]$.

Применяя равенство (24) к пробной функции $w(t, x, v)$ получим, что

$$
\begin{aligned}
\int_{\Pi \times \mathbb{R}} f(t, x, v)\left[w_{t}(t, x, v)\right. & \left.+\left(\varphi^{\prime}(v), \nabla_{x} w\right)\right] d t d x d v \\
& =-r \int_{\Pi \times \mathbb{R}}(g(t, x, v)-f(t, x, v)) w(t, x, v) d t d x d v
\end{aligned}
$$

или, раскрыв производные $w_{t}, \nabla_{x} w$,

$$
\begin{aligned}
& \int_{0}^{+\infty}\left(\int_{\mathbb{R}^{n+1}} f(t, x, v) \beta_{h}(x, v) \rho(x) \xi(v) d x d v\right)\left(\delta_{\nu}\left(t-t_{0}\right)-\delta_{\nu}\left(t-t_{1}\right)\right) d t \\
& \quad+\int_{\Pi \times \mathbb{R}} f(t, x, v)\left(\varphi^{\prime}(v), \nabla_{x}\left(\beta_{h}(x, v) \rho(x)\right)\right) \xi(v) \chi_{\nu}(t) d t d x d v \\
& \quad=-r \int_{\Pi}\left(\int_{\mathbb{R}}(g(t, x, v)-f(t, x, v)) \beta_{h}(x, v) \xi(v) d v\right) \rho(x) \chi_{\nu}(t) d t d x
\end{aligned}
$$


Так как $f_{0}(x, v)$ не возрастает по переменной $v \in \mathbb{R}$, то функция $\beta_{h}(x, v)$ также не возрастает по $v$, и по свойству 1) из леммы 4

$\int_{\mathbb{R}}(g(t, x, v)-f(t, x, v)) \beta_{h}(x, v) \xi(v) d v=\int_{-M}^{M}(g(t, x, v)-f(t, x, v)) \beta_{h}(x, v) d v \geqslant 0$

на множестве полной меры значений $(t, x) \in \Pi$, при которых функция $v \mapsto f(t, x, v)$ измерима и в соответствии с замечанием $1 g(t, x, v)=f^{*}(t, x ; v)$. Поэтому правая часть (39) неположительна и, значит,

$$
\begin{aligned}
& \int_{0}^{+\infty}\left(\int_{\mathbb{R}^{n+1}} f(t, x, v) \beta_{h}(x, v) \rho(x) \xi(v) d x d v\right) \delta_{\nu}\left(t-t_{0}\right) d t \\
& \quad \leqslant \int_{0}^{+\infty}\left(\int_{\mathbb{R}^{n+1}} f(t, x, v) \beta_{h}(x, v) \rho(x) \xi(v) d x d v\right) \delta_{\nu}\left(t-t_{1}\right) d t \\
& \quad+\left|\int_{\Pi \times \mathbb{R}} f(t, x, v)\left(\varphi^{\prime}(v), \nabla_{x}\left(\beta_{h}(x, v) \rho(x)\right)\right) \xi(v) \chi_{\nu}(t) d t d x d v\right| .
\end{aligned}
$$

Как следует из равенства (33), o.p. $f(t, x, v)$ (может быть, после "исправления" на множестве нулевой меры) непрерывно по $t \in[0,+\infty)$ в $L^{\infty}\left(\mathbb{R}^{n+1}\right)$. В частности, можно считать, что функции

$$
t \mapsto \int_{\mathbb{R}^{n+1}} f(t, x, v) \beta_{h}(x, v) \rho(x) \xi(v) d x d v
$$

непрерывны по $t$ и из (40) в пределе при $\nu \rightarrow \infty$ вытекает оценка

$$
\begin{aligned}
& \int_{\mathbb{R}^{n+1}} f\left(t_{0}, x, v\right) \beta_{h}(x, v) \rho(x) \xi(v) d x d v \\
& \quad \leqslant \int_{\mathbb{R}^{n+1}} f\left(t_{1}, x, v\right) \beta_{h}(x, v) \rho(x) \xi(v) d x d v \\
& \quad+\int_{t_{0}}^{t_{1}} \int_{\mathbb{R}^{n+1}}\left|\left(\varphi^{\prime}(v), \nabla_{x}\left(\beta_{h}(x, v) \rho(x)\right)\right)\right| f(t, x, v) \xi(v) d t d x d v .
\end{aligned}
$$

Далее, при $h<1$ с учетом оценки (38)

$$
\begin{aligned}
\nabla_{x}\left(\beta_{h}(x, v) \rho(x)\right) & \leqslant\left|\nabla_{x} \beta_{h}(x, v)\right| \rho(x)+\beta_{h}\left|\nabla_{x} \rho(x)\right| \\
& \leqslant \frac{c_{1}}{h} \rho(x)+\rho(x) \leqslant \frac{c_{2}}{h} \rho(x), \quad c_{2}=c_{1}+1
\end{aligned}
$$

(заметим, что $\left|\nabla_{x} \rho(x)\right|=\rho(x)$ и, поскольку $0 \leqslant f_{0}(x, v) \leqslant 1$, то и $0 \leqslant \beta_{h}(x, v) \leqslant 1$ ). Поэтому, из (41) и из того, что $f\left(t_{0}, x, v\right)-f\left(t_{1}, x, v\right)=0$ для п.в. $(x, v) \in \mathbb{R}^{n} \times$ $(\mathbb{R} \backslash[-M, M])$

$$
\begin{aligned}
\int_{\mathbb{R}^{n+1}} & \left(f\left(t_{0}, x, v\right)-f\left(t_{1}, x, v\right)\right) \beta_{h}(x, v) \rho(x) d x d v \\
& \leqslant \int_{t_{0}}^{t_{1}} \int_{\mathbb{R}^{n+1}}\left|\varphi^{\prime}(v)\right|\left|\nabla_{x}\left(\beta_{h} \rho\right)\right| f(t, x, v) \xi(v) d t d x d v \leqslant \frac{c_{3}}{h}\left(t_{1}-t_{0}\right),
\end{aligned}
$$




$$
\begin{gathered}
c_{3}=2 M N c_{2} \int_{\mathbb{R}^{n}} \rho(x) d x, \text { где обозначено } N=\max _{v \in[-M, M]}\left|\varphi^{\prime}(v)\right| . \text { Далее, } \\
\int_{\mathbb{R}^{n+1}}\left|\beta_{h}(x, v)-f_{0}(x, v)\right| \rho(x) d x d v \\
\quad=\int_{\mathbb{R}^{n+1}}\left|\int_{\mathbb{R}^{n}}\left(f_{0}(x-y, v)-f_{0}(x, v)\right) \gamma_{h}(y) d y\right| \rho(x) d x d v \\
\leqslant \int_{\mathbb{R}^{n}}\left(\int_{\mathbb{R}^{n+1}}\left|f_{0}(x-y, v)-f_{0}(x, v)\right| \rho(x) d x d v\right) \gamma_{h}(y) d y \leqslant \omega_{1}^{x}(h),
\end{gathered}
$$

так как

$$
\operatorname{supp} \gamma_{h} \subset B_{h} \quad \text { и } \int_{\mathbb{R}^{n}} \gamma_{h}(y) d y=1 .
$$

С учетом (42), (43) и оценки $\left\|f\left(t_{0}, \cdot\right)-f\left(t_{1}, \cdot\right)\right\|_{\infty} \leqslant 1$

$$
\begin{aligned}
& \int_{\mathbb{R}^{n+1}}\left(f\left(t_{0}, x, v\right)-f\left(t_{1}, x, v\right)\right) f_{0}(x, v) \rho(x) d x d v \\
& \quad \leqslant \int_{\mathbb{R}^{n+1}}\left(f\left(t_{0}, x, v\right)-f\left(t_{1}, x, v\right)\right)\left(f_{0}(x, v)-\beta_{h}(x, v)\right) \rho(x) d x d v \\
& \quad+\int_{\mathbb{R}^{n+1}}\left(f\left(t_{0}, x, v\right)-f\left(t_{1}, x, v\right)\right) \beta_{h}(x, v) \rho(x) d x d v \\
& \quad \leqslant \omega_{1}^{x}(h)+\frac{c_{3}}{h}\left(t_{1}-t_{0}\right) .
\end{aligned}
$$

Переходя к пределу при $t_{0} \rightarrow 0$, получим из (44) (для удобства мы меняем обозначение $t_{1}$ на $\Delta t$ ), что $\forall \Delta t>0$

$$
\int_{\mathbb{R}^{n+1}}\left(f_{0}(x, v)-f(\Delta t, x, v)\right) f_{0}(x, v) \rho(x) d x d v \leqslant \omega_{1}^{x}(h)+\frac{c_{3}}{h} \Delta t
$$

Далее, умножим $(24)$ на $2 f(t, x, v)$. Получим равенство

$$
\begin{aligned}
\frac{\partial}{\partial t} f^{2}(t, x, v)+\left(\varphi^{\prime}(v), \nabla_{x}\right. & \left.f^{2}(t, x, v)\right) \\
& =2 r(g(t, x, v)-f(t, x, v)) f(t, x, v) \quad \text { в } \quad D^{\prime}(\Pi \times \mathbb{R}),
\end{aligned}
$$

применяя которое к пробной функции $\rho(x) \chi_{\nu}(t) \xi(v)$, где $\chi_{\nu}(t)=\alpha_{\nu}\left(t-t_{0}\right)-$ $\alpha_{\nu}\left(t-t_{1}\right), 0<t_{0}<t_{1}, \nu \in \mathbb{N}$, получим, после элементарных преобразований, что

$$
\begin{aligned}
& \int_{\Pi \times \mathbb{R}} f^{2}(t, x, v) \rho(x) \xi(v)\left(\delta_{\nu}\left(t-t_{0}\right)-\delta_{\nu}\left(t-t_{1}\right)\right) d t d x d v \\
& +\int_{\Pi \times \mathbb{R}}\left(\varphi^{\prime}(v), \nabla_{x} \rho(x)\right) f^{2}(t, x, v) \xi(v) \chi_{\nu}(t) d t d x d v \\
& \quad=-2 r \int_{\Pi}\left(\int_{-M}^{M} g(t, x, v) f(t, x, v) d v-\int_{-M}^{M} f^{2}(t, x, v) d v\right) \rho(x) \chi_{\nu}(t) d t d x
\end{aligned}
$$


Здесь с учетом замечания $1 g(t, x, v)=f^{*}(t, x, v)$ при $v \in(-M, M)$. По неравенству Гёльдера и равенству (22) для п.в. $(t, x) \in \Pi$

$$
\begin{aligned}
\int_{-M}^{M} g(t, x, v) f(t, x, v) d v & =\int_{-M}^{M} f^{*}(t, x, v) f(t, x, v) d v \\
& \leqslant\left(\int_{-M}^{M}\left(f^{*}(t, x, v)\right)^{2} d v\right)^{1 / 2}\left(\int_{-M}^{M}(f(t, x, v))^{2} d v\right)^{1 / 2} \\
& =\int_{-M}^{M}(f(t, x, v))^{2} d v .
\end{aligned}
$$

Поэтому правая часть (46) неотрицательна и из (46)

$$
\begin{aligned}
\int_{\Pi \times \mathbb{R}} f^{2}(t, x, v) \rho(x) \xi(v) & \left(\delta_{\nu}\left(t-t_{1}\right)-\delta_{\nu}\left(t-t_{0}\right)\right) d t d x d v \\
\leqslant & \int_{\Pi \times \mathbb{R}}\left|\left(\varphi^{\prime}(v), \nabla_{x} \rho(x)\right)\right| f^{2}(t, x, v) \xi(v) \chi_{\nu}(t) d t d x d v .
\end{aligned}
$$

Отсюда с учетом оценок $\left|\left(\varphi^{\prime}(v), \nabla_{x} \rho(x)\right)\right| \leqslant\left|\varphi^{\prime}(v)\right|\left|\nabla_{x} \rho(x)\right| \leqslant N \rho(x)$ при $v \in$ $[-M, M],\|f(t, x, v)\|_{\infty} \leqslant 1$ получаем, что

$$
\begin{gathered}
\int\left(\int_{\mathbb{R}^{n+1}} f^{2}(t, x, v) \rho(x) \xi(v) d t d x d v\right)\left(\delta_{\nu}\left(t-t_{1}\right)-\delta_{\nu}\left(t-t_{0}\right)\right) d t \leqslant c_{4} \int \chi_{\nu}(t) d t, \\
c_{4}=\text { const } .
\end{gathered}
$$

Как отмечено вьше, можно считать, что отображение $t \mapsto f(t, x, v)$ непрерывно в $L^{\infty}\left(\mathbb{R}^{n+1}\right)$, что влечет непрерывность функции $t \mapsto \int_{\mathbb{R}^{n+1}} f^{2}(t, x, v) \rho(x) \xi(v) d x d v$. Следовательно, в пределе при $\nu \rightarrow \infty$

$$
\int_{\mathbb{R}^{n+1}}\left(f^{2}\left(t_{1}, x, v\right)-f^{2}\left(t_{0}, x, v\right)\right) \rho(x) d x d v \leqslant c_{4}\left(t_{1}-t_{0}\right) .
$$

Переходя в этой оценке к пределу при $t_{0} \rightarrow 0$ (с учетом $\left.(25)\right)$ и заменяя $t_{1}$ на $\Delta t$, получим, что $\forall \Delta t>0$

$$
\int_{\mathbb{R}^{n+1}}\left(f^{2}(\Delta t, x, v)-f_{0}^{2}(x, v)\right) \rho(x) d x d v \leqslant c_{4} \Delta t .
$$

Наконец, ввиду (45), (47)

$$
\begin{aligned}
& \int_{\mathbb{R}^{n+1}}\left(f(\Delta t, x, v)-f_{0}(x, v)\right)^{2} \rho(x) d x d v \\
& =\int_{\mathbb{R}^{n+1}}\left(f^{2}(\Delta t, x, v)-f_{0}^{2}(x, v)\right) \rho(x) d x d v \\
& \quad+2 \int_{\mathbb{R}^{n+1}}\left(f_{0}(x, v)-f(\Delta t, x, v)\right) f_{0}(x, v) \rho(x) d x d v \\
& \quad \leqslant c_{4} \Delta t+2\left(\frac{c_{3} \Delta t}{h}+\omega_{1}^{x}(h)\right) \leqslant c\left(\omega_{1}^{x}(h)+\frac{\Delta t}{h}\right), \quad c=2 c_{3}+c_{4}+2,
\end{aligned}
$$

откуда в силу произвольности $h \in(0,1)$ следует требуемая оценка. Теорема доказана.

В дополнение к теореме заметим, что $\omega^{t}(\Delta t) \rightarrow 0$ при $\Delta t \rightarrow 0$. Действительно, взяв $h=\sqrt{\Delta t}$ при $\Delta t<1$, получим, что $\omega^{t}(\Delta t) \leqslant c\left(\omega_{1}^{x}(\sqrt{\Delta t})+\sqrt{\Delta t}\right)$. 
СлЕДСТвИЕ 3. Для n.в. $t_{1}, t_{0} \in(0, \infty), t_{1}>t_{0}$,

$$
\int_{\mathbb{R}^{n+1}}\left(f\left(t_{1}, x, v\right)-f\left(t_{0}, x, v\right)\right)^{2} \rho(x) d x d v \leqslant \omega^{t}(\Delta t), \quad \Delta t=\left(t_{1}-t_{0}\right) .
$$

ДокАЗАТЕЛЬСТво. Можно считать (см. доказательство теоремы 4), что отображение $t \mapsto f(t, x, v)$ непрерывно при $t \in[0,+\infty)$ в $L^{\infty}\left(\mathbb{R}^{n+1}\right)$. Тогда при $\Delta t>0$ функции $f_{1}(t, x, v)=f(t, x, v), f_{2}(t, x, v)=f(t+\Delta t, x, v)$ являются о.р. задачи $(24),(25)$ с начальными функциями $f_{0}(x, v), f(\Delta t, x, v)$, соответственно. Тогда по теореме 2 для $t \in(0,+\infty)$

$$
\begin{aligned}
\int_{\mathbb{R}^{n+1}}(f(t+\Delta t, x, v)- & f(t, x, v))^{2} \rho(x) d x d v \\
\leqslant & \int_{\mathbb{R}^{n+1}}\left(f(\Delta t, x, v)-f_{0}(x, v)\right)^{2} \rho(x) d x d v \leqslant \omega^{t}(\Delta t),
\end{aligned}
$$

что и нужно было доказать.

\section{§3. Сходимость аппроксимаций}

Пусть $f_{r}(t, x, v), r \in \mathbb{N},-$ последовательность о.р. задачи $(24),(25)$

$$
f_{t}+\left(\varphi^{\prime}(v), \nabla_{x} f\right)=r(g-f), \quad g=F f ; \quad f(0, x, v)=f_{0}(x, v) \in F_{M}\left(\mathbb{R}^{n}\right) .
$$

Покажем, что эта последовательность (может быть, после выделения подпоследовательности) сходится в $L_{\text {loc }}^{1}(\Pi \times \mathbb{R})$ к о.p. $f(t, x, v)$ задачи $(4),(5)$.

Нам понадобится

Лемма 6. Пусть $g_{r}=F f_{r}$. Тогда для некоторой неотрицательной локально конечной борелевской меры $m_{r}$ на $\Pi \times[-M, M]$

$$
r\left(g_{r}(t, x, v)-f_{r}(t, x, v)\right)=\frac{\partial}{\partial v} m_{r}(t, x, v) \quad \text { в } \quad D^{\prime}(\Pi \times \mathbb{R}) .
$$

При этом для любого компакта $C \subset \Pi$ значения $m_{r}(C \times \mathbb{R})$ ограничены по $r \in \mathbb{N}$.

ДокАЗАТЕльство. Пусть $\rho_{r}(t, x, v)=r \int_{-M}^{v}\left(g_{r}(t, x, \lambda)-f_{r}(t, x, \lambda)\right) d \lambda$ для множества полной меры значений $(t, x) \in \Pi$, при которых последний интеграл определен. По свойству 1$)$ из леммы $4 \rho_{r}(t, x, v) \geqslant 0$ и, очевидно,

$$
r\left(g_{r}-f_{r}\right)=\frac{\partial}{\partial v} \rho_{r}=\frac{\partial}{\partial v} m_{r} \quad \text { в } D^{\prime}(\Pi \times \mathbb{R}),
$$

где $m_{r}$ - неотрицательная мера: $m_{r}(t, x, v)=\rho_{r}(t, x, v) d t d x d v$ на $\Pi \times \mathbb{R}$. Так как $g_{r}(t, x, v)-f_{r}(t, x, v)=0$ п.в. на $\Pi \times(\mathbb{R} \backslash[-M, M])$ и

$$
\int_{-M}^{M}\left(g_{r}(t, x, \lambda)-f_{r}(t, x, \lambda)\right) d \lambda=\int_{-M}^{M}\left(f_{r}^{*}(t, x ; \lambda)-f_{r}(t, x, \lambda)\right) d \lambda=0
$$


по равенству $(22)$, то $\rho_{r}(t, x, v)=0$ п.в. на $\Pi \times(\mathbb{R} \backslash[-M, M])$, откуда следует включение $\operatorname{supp} m_{r} \subset \Pi \times[-M, M]$. Пусть далее $C \subset \Pi-$ компакт, $h(t, x) \in C_{0}^{\infty}(\Pi), h(t, x) \geqslant 0, h(t, x) \equiv 1$ на $C ; \chi(v) \in C_{0}^{\infty}(\mathbb{R}), \quad \chi^{\prime}(v)=1$ на $[-M, M]$. Применяя равенство

$$
\frac{\partial f_{r}}{\partial t}+\left(\varphi^{\prime}(v), \nabla_{x} f_{r}\right)=r\left(g_{r}-f_{r}\right)=\frac{\partial}{\partial v} m_{r}
$$

к пробной функции $h(t, x) \chi(v)$, получим оценку

$$
\begin{aligned}
m_{r}(C \times[-M, M]) & \leqslant \int_{\Pi \times \mathbb{R}} h(t, x) \chi^{\prime}(v) d m_{r}(t, x, v) \\
& =\int_{\Pi \times \mathbb{R}} f_{r}(t, x, v)\left[h_{t}+\left(\varphi^{\prime}(v), \nabla_{x} h\right)\right] \chi(v) d t d x d v \\
& \leqslant c=\left\|\left(h_{t}+\left(\varphi^{\prime}(v), \nabla_{x} h\right)\right) \chi(v)\right\|_{L^{1}(\Pi \times \mathbb{R})} .
\end{aligned}
$$

Мы учли, что по лемме $5\left\|f_{r}\right\|_{\infty} \leqslant 1$. Итак, значения $m_{r}(C \times[-M, M])=$ $m_{r}(C \times \mathbb{R})$ ограничены по $r \in \mathbb{N}$. Лемма доказана.

ТЕОРема 5. Существует подпоследовательность последовательности $f_{r}(t, x, v)$, сходящаяся в $L_{\mathrm{loc}}^{1}(\Pi \times \mathbb{R}) \kappa$ о.p. $f(t, x, v) \in F_{M}(\Pi)$ задачи (4), (5).

ДоказАтЕльство. Так как по лемме 5 для всех $r \in \mathbb{N} 0 \leqslant f_{r}(t, x, v) \leqslant 1$ п.в. на $\Pi \times \mathbb{R}$ и по лемме 6 последовательность мер $m_{r}$ ограничена по вариации на любом компакте, то, после выделения подпоследовательности (за которой мы сохраним прежнее обозначение) при $r \rightarrow \infty f_{r}(t, x, v) \rightarrow f(t, x, v) \quad *$-слабо в $L^{\infty}(\Pi \times \mathbb{R})$, $m_{r} \rightarrow m$ слабо в пространстве $Z_{\text {lос }}(\Pi \times \mathbb{R})$ локально конечных зарядов на $\Pi \times \mathbb{R}$, где $f(t, x, v) \in L^{\infty}(\Pi \times \mathbb{R}), m \in Z_{\mathrm{loc}}(\Pi \times \mathbb{R}), m=m(t, x, v) \geqslant 0, \operatorname{supp} m \subset \Pi \times[-M, M]$. В пределе при $r \rightarrow \infty$ из равенства

$$
\frac{\partial}{\partial t} f_{r}+\left(\varphi^{\prime}(v), \nabla_{x} f_{r}\right)=r\left(g_{r}-f_{r}\right)=\frac{\partial}{\partial v} m_{r} \quad \text { в } \quad D^{\prime}(\Pi \times \mathbb{R})
$$

следует, что

$$
\frac{\partial}{\partial t} f+\left(\varphi^{\prime}(v), \nabla_{x} f\right)=\frac{\partial}{\partial v} m \quad \text { в } \quad D^{\prime}(\Pi \times \mathbb{R}),
$$

т.е. $f=f(t, x, v)$ удовлетворяет кинетическому уравнению (4). Далее, по равенству $(22)$ с $p(u)=u^{2}$

$$
\int_{\mathbb{R}}\left(g_{r}-f_{r}\right)\left(g_{r}+f_{r}\right) d v=\int_{\mathbb{R}}\left(g_{r}^{2}-f_{r}^{2}\right) d v=0,
$$

откуда следует, что

$$
\int_{\mathbb{R}}\left(g_{r}-f_{r}\right) g_{r} d v=-\int_{\mathbb{R}}\left(g_{r}-f_{r}\right) f_{r} d v
$$

и поэтому

$$
\int_{\mathbb{R}}\left(g_{r}-f_{r}\right)^{2} d v=\int_{\mathbb{R}}\left(g_{r}-f_{r}\right) g_{r} d v-\int_{\mathbb{R}}\left(g_{r}-f_{r}\right) f_{r} d v=-2 \int_{\mathbb{R}}\left(g_{r}-f_{r}\right) f_{r} d v
$$


Умножим это равенство на неотрицательную функцию $h=h(t, x) \in C_{0}^{1}(\Pi)$ и проинтегрируем по $(t, x) \in$ П. Получим, что

$$
\int_{\Pi \times \mathbb{R}}\left(g_{r}-f_{r}\right)^{2} h d t d x d v=-2 \int_{\Pi \times \mathbb{R}}\left(g_{r}-f_{r}\right) f_{r} h d t d x d v,
$$

откуда в силу соотношения

$$
\frac{\partial}{\partial t} f_{r}^{2}+\left(\varphi^{\prime}(v), \nabla_{x} f_{r}^{2}\right)=2 r\left(g_{r}-f_{r}\right) f_{r} \quad \text { в } \quad D^{\prime}(\Pi \times \mathbb{R})
$$

следует равенство (в котором $\xi(v)$ - характеристическая функция интервала $[-M, M])$

$$
\begin{aligned}
\int_{\Pi \times \mathbb{R}}\left(g_{r}-f_{r}\right)^{2} h d t d x d v & =-\frac{1}{r}\left\langle\frac{\partial}{\partial t} f_{r}^{2}+\left(\varphi^{\prime}(v), \nabla_{x} f_{r}^{2}\right), h(t, x) \xi(v)\right\rangle \\
& =\frac{1}{r} \int_{\Pi \times \mathbb{R}} f_{r}^{2}\left[h_{t}+\left(\varphi^{\prime}(v), \nabla_{x} h\right)\right] \xi(v) d t d x d v \leqslant \frac{c}{r} \\
c=\mathrm{const} & =\left\|\left(h_{t}+\left(\varphi^{\prime}(v), \nabla_{x} h\right)\right) \xi(v)\right\|_{L^{1}(\Pi \times \mathbb{R})} .
\end{aligned}
$$

Из полученной оценки следует предельное соотношение

$$
\int_{\Pi \times \mathbb{R}}\left|g_{r}-f_{r}\right| h d t d x d v \leqslant \int_{\Pi \times \mathbb{R}}\left(g_{r}-f_{r}\right)^{2} h d t d x d v \underset{r \rightarrow \infty}{\longrightarrow} 0
$$

и ввиду произвольности неотрицательной функции $h \in C_{0}^{1}($ П) при $r \rightarrow \infty$

$$
g_{r}(t, x, v)-f_{r}(t, x, v) \rightarrow 0 \quad \text { в } \quad L_{\mathrm{loc}}^{1}(\Pi \times \mathbb{R}) .
$$

Из (48), в частности, следует, что при $r \rightarrow \infty$ последовательность $g_{r}(t, x, v)$ сходится к $f(t, x, v) *$-слабо в $L^{\infty}(\Pi \times \mathbb{R})$ (одновременно с последовательностью $f_{r}$ ).

Так как функции $g_{r}(t, x, v)$ не возрастают по переменной $v$, то после возможного "исправления" предельной функции $f(t, x, v)$ на множестве нулевой меры $f(t, x, v)$ также не возрастает и непрерывна справа по переменной $v$. Ясно также, что $0 \leqslant f(t, x, v) \leqslant 1$ п.в. на $\Pi \times \mathbb{R}, f(t, x, v)=1$ п.в. на $\Pi \times(-\infty,-M), f(t, x, v)=0$ п.в. на $\Pi \times(M,+\infty)$. Таким образом, $f(t, x, v) \in F_{M}(\Pi)$.

Пусть, далее, $\chi(v) \in C_{0}^{1}(\mathbb{R})$,

$$
p_{r}(t, x)=\int f_{r}(t, x, v) \chi(v) d v, \quad q_{r}(t, x)=\int g_{r}(t, x, v) \chi(v) d v .
$$

Ясно, что при $r \rightarrow \infty$

$$
p_{r}(t, x) \rightarrow p(t, x), \quad q_{r}(t, x) \rightarrow p(t, x) \quad * \text {-слабо в } L^{\infty}(\Pi),
$$

где $p(t, x)=\int f(t, x, v) \chi(v) d v$.

Из оценок, содержащихся в следствиях 2,3 , вытекает равностепенная непрерывность последовательности $p_{r}(t, x)$ в $L^{1}\left(\Pi_{T}, \rho(x) d t d x\right), T>0$ произвольно. По 
критерию Хаусдорфа, сходимость в (49) сильная - в $L_{\text {loc }}^{1}($ П) (для последовательности $q_{r}$ нужно учесть (48)).

Выберем счетное всюду плотное множество $S \subset C_{0}^{1}(\mathbb{R})$. Тогда, применяя при дополнительном выделении подпоследовательности стандартную диагональную процедуру, можем считать, что для всех $\chi(v) \in S$

$$
q_{r}(t, x)=\int g_{r}(t, x, v) \chi(v) d v \underset{r \rightarrow \infty}{\longrightarrow} \int f(t, x, v) \chi(v) d v
$$

в $L_{\text {loc }}^{1}(\Pi)$ и п.в. на П. Из плотности $S$ в $C_{0}^{1}(\mathbb{R})\left(\right.$ и, значит, в $\left.L^{1}(\mathbb{R})\right)$, для п.в. $(t, x) \in \Pi$

$$
g_{r}(t, x, v) \underset{r \rightarrow \infty}{\longrightarrow} f(t, x, v)
$$

по $v \in \mathbb{R} *$-слабо в $L^{\infty}(\mathbb{R})$.

Так как функции $g_{r}(t, x, v)$ монотонны по $v$, то по теореме Хелли (см. [11, c. 209]) $g_{r}(t, x, v) \underset{r \rightarrow \infty}{\longrightarrow} f(t, x, v)$ во всех точках непрерывности функции $v \mapsto f(t, x, v)$. Дополнение ко множеству точек непрерывности в силу монотонности не более чем счетно. Итак, измеримое множество $\left\{(t, x, v) \in \Pi \times \mathbb{R} \mid g_{r}(t, x, v) \underset{r \rightarrow \infty}{\longrightarrow} f(t, x, v)\right\}$ имеет полную меру.

Значит, $g_{r}(t, x, v) \underset{r \rightarrow \infty}{\longrightarrow} f(t, x, v)$ п.в. на $\Pi \times \mathbb{R}$ и (с учетом ограниченности $\left.g_{r}\right)$ в $L_{\text {lос }}^{1}(\Pi \times \mathbb{R})$. Из $(48)$ следует, что последовательность $f_{r}(t, x, v)$ также сходится к $f(t, x, v)$ в $L_{\text {loc }}^{1}(\Pi \times \mathbb{R})$.

Для завершения доказательства осталось проверить выполнение начального условия (26) для предельной функции $f(t, x, v)$. По теореме 4 для п.в. $\Delta t>0$

$$
\int_{\mathbb{R}^{n+1}}\left(f_{r}(\Delta t, x, v)-f_{0}(x, v)\right)^{2} \rho(x) d x d v \leqslant \omega^{t}(\Delta t),
$$

$\omega^{t}(\Delta t)$ не зависит от $r$.

Дляп.в. $\Delta t>0$ 0последовательность $f_{r}(\Delta t, \cdot)-f_{0}$ сходитсяв $L^{1}\left(\mathbb{R}^{n+1}, \rho(x) d x d v\right)$ к функции $f(\Delta t, \cdot)-f_{0}$ и из $(50)$ в пределе при $r \rightarrow \infty$ вытекает, что для п.в. $\Delta t>0$

$$
\int_{\mathbb{R}^{n+1}}\left(f(\Delta t, x, v)-f_{0}(x, v)\right)^{2} \rho(x) d x d v \leqslant \omega^{t}(\Delta t) \underset{\Delta t \rightarrow 0}{\longrightarrow} 0 .
$$

Итак,

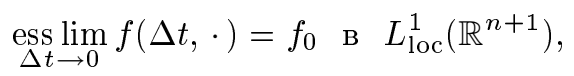

т.е. вьполнено начальное условие $(25)$ и $f(t, x, v)$ - о.р. задачи $(24),(25)$. Теорема полностью доказана.

Предположим, далее, что $\nu_{x}^{0}(\lambda)=\delta\left(\lambda-u_{0}(x)\right)$ - регулярная начальная функция. Известно (см. [6], [7], [9]), что в этом случае м.р. $\nu_{t, x}$ задачи Коши $(1),(7)$ также регулярно и единственно: $\nu_{t, x}=\delta(\lambda-u(t, x))$, где $u(t, x)$ - единственное o.p. задачи $(1),(2)$ с начальной функцией $u_{0}(x)$. Легко видеть, что соответствующая функция распределения $f(t, x, v)=\nu_{t, x}((v,+\infty))=\theta(u(t, x)-v)$, где $\theta(u)$ - функция Хевисайда. Из единственности решения $\nu_{t, x}$ следует, что предельная функция $f(t, x, v)$ в теореме 5 не зависит от выбора подпоследовательности. Таким образом, $f(t, x, v)$ - единственная предельная точка последовательности $f_{r}(t, x, v)$, $r \in \mathbb{N}$, в $L_{\text {loc }}^{1}(\Pi \times \mathbb{R})$. Это означает, что исходная последовательность $f_{r}(t, x, v)$ сходится к $f(t, x, v)$ в $L_{\text {loc }}^{1}(\Pi \times \mathbb{R})$ (без выделения подпоследовательности). 


\section{Список литературы}

1. Кружков C.H. Обобщенные решения задачи Коши в целом для нелинейных уравнений первого порядка // ДАН СССР. 1969. Т. 187. № 1. С. 29-32.

2. Кружкков C. H. Квазилинейные уравнения первого порядка со многими независимыми переменными // Матем. сб. 1970. Т. 81. № 2. С. 228-255.

3. Lions P. L., Perthame B., Tadmor E. A kinetic formulation of multidimensional scalar conservation laws and related equations // J. Amer. Math. Soc. 1994. V. 7. № 1. P. 169-191.

4. Tartar $L$. Compensated compactness and applications to partial differential equations // Research notes in mathematics, nonlinear analysis, and mechanics. Heriot-Watt Symposium. V. 4, 1979. P. 136-212.

5. DiPerna R. J. Generalized solutions to conservation laws, in system of nonlinear partial differential equations // NATO ASI Series / ed. J.M. Ball: D. Reidel Pub. Co., 1983.

6. DiPerna R.J. Measure-valued solutions to conservation laws // Arch. Rational Mech. Anal. 1985. V. 88. P. 223-270.

7. Панов Е. Ю. Обобщенные решения задачи Коши для квазилинейных законов сохранения // Дис. ... канд. физ.-матем. наук. М: МГУ, 1991.

8. Панов Е. Ю. Сильные мерозначные решения задачи Коши для квазилинейного уравнения первого порядка с ограниченной мерозначной начальной функцией // Вестн. МГУ. Сер. 1. Матем, мех. 1993. №1. С. 20-23.

9. Панов E. Ю. О мерозначных решениях задачи Коши для квазилинейного уравнения первого порядка // Изв. РАН. Сер. матем. 1996. Т. 60. № 2. С. 107-148.

10. Панов $E$. Ю. О последовательностях мерозначных решений квазилинейного уравнения первого порядка // Матем. сб. 1994. Т. 185. № 2. С. 87-106.

11. Натансон И. П. Теория функций вещественной переменной. М.: Гостехиздат, 1957.

12. Харди Г. Г., Литтлвуд Дж. Е., Полиа Г. Неравенства. М.: ИЛ, 1948.

Новгородский государственный университет

Поступила в редакцию

06.02 .1996 